\title{
Synthesis of Multi(metallo)porphyrin Dendrimers through Nucleophilic Aromatic Substitution on meso-Pyrimidinyl Substituted Porphyrins
}

\author{
Wouter Maes, ${ }^{\dagger}$ Jeroen Vanderhaeghen, ${ }^{\dagger}$ Stefan Smeets, ${ }^{\dagger}$ C.V. Asokan, ${ }^{\ddagger}$ Lieven M. Van Renterghem, ${ }^{\S}$ \\ Filip E. Du Prez, ${ }^{\S}$ Mario Smet ${ }^{\dagger}$ and Wim Dehaen ${ }^{*, \dagger}$ \\ ${ }^{\dagger}$ Molecular Design and Synthesis, Department of Chemistry, Katholieke Universiteit Leuven, \\ Celestijnenlaan 200F, B-3001 Leuven, Belgium \\ ${ }^{\ddagger}$ School of Chemical Sciences, Mahatma Gandhi University, India 686560 \\ $\S$ Department of Organic Chemistry, Polymer Chemistry Research Group, Ghent University, Krijgslaan \\ 281 S4-bis, B-9000 Ghent, Belgium \\ *E-mail: wim.dehaen@chem.kuleuven.be; Fax: +3216327990; Tel. +3216327390
}

\section{Table of Contents}

1. General Experimental Methods

3. Selected NMR Spectra: porphyrin monomer $4 \mathbf{a}\left({ }^{1} \mathrm{H}\right.$ and $\left.{ }^{13} \mathrm{C}\right), \mathrm{G}_{1}$-dendron $\mathbf{5 b}\left({ }^{1} \mathrm{H},{ }^{13} \mathrm{C}\right.$ and $2 \mathrm{D}$ NMR), $\mathrm{G}_{2}$-dendrons 7b,c $\left({ }^{1} \mathrm{H}\right)$ and $\mathrm{G}_{0}$-dendrimer 11a $\left({ }^{1} \mathrm{H}\right)$

4. Selected MS Spectra: $\mathrm{G}_{1}$-dendron 5b (ESI), $\mathrm{G}_{2}$-dendron 7c (MALDI), $\mathrm{G}_{0}$-dendrimer 11a (ESI) and $\mathrm{G}_{1}$-dendrimer 12 (MALDI) 


\section{General Experimental Methods}

NMR chemical shifts $(\delta)$ are reported in parts per million referenced to tetramethylsilane (TMS) $\left({ }^{1} \mathrm{H}\right)$ or the carbon signal of deuterated solvents $\left({ }^{13} \mathrm{C}\right)$. Detailed ${ }^{13} \mathrm{C}$ NMR peak assignments were obtained by careful analysis of DEPT, HMQC and HMBC NMR spectra. For some of the higher generation dendrons and dendrimers complete ${ }^{13} \mathrm{C}$ assignment could not be accomplished due to the low abundance of some (interior) signals. Electrospray (ESI) mass spectra were run using a solvent mixture $\mathrm{CH}_{2} \mathrm{Cl}_{2} / \mathrm{MeOH}+\mathrm{NH}_{4} \mathrm{OAc}$. MALDI-TOF mass spectra were obtained with trans-3-indoleacrylic acid (IAA) (in THF) or trans-2-[3-(4-tert-butylphenyl)-2-methyl-2-propenylidene]malononitrile (BMPM) (in THF) as a matrix. Analytical gel permeation chromatography (GPC) was carried out using a Plgel mixed-D column (Polymer Laboratories): RI and UV detector, $\mathrm{CHCl}_{3}$ as the eluent at a flow of $1 \mathrm{~mL}$ $\min ^{-1}$ and at $30{ }^{\circ} \mathrm{C}$, calibration with linear polystyrene standards. For column chromatography 70-230 mesh silica 60 (E.M. Merck) was used as the stationary phase. Chemicals received from commercial sources were used without further purification. DMF was dried on molecular sieves $4 \AA$. 


\section{Experimental and Characterization Data}

\section{Zinc(II) 5-(4-Benzyloxyphenyl)-15-(4,6-dichloropyrimidin-5-yl)-10,20-bis(2,4,6-trimethylphe-}

nyl)porphyrin (4b). Metallation procedure: cfr. 3c (100 mg scale). MS (ESI) calcd for $\mathrm{C}_{55} \mathrm{H}_{42} \mathrm{Cl}_{2} \mathrm{~N}_{6} \mathrm{OZn}$ : 939.3; found: $\mathrm{m} / z$ 938.1; ${ }^{1} \mathrm{H} \mathrm{NMR}\left(300 \mathrm{MHz}, \mathrm{CDCl}_{3}\right) \delta 8.91\left(\mathrm{~d},{ }^{3} \mathrm{~J}=4.8 \mathrm{~Hz}, 2 \mathrm{H}, \mathrm{H}_{\beta^{-}}\right.$ Zn-porph.), 8.79 (d, ${ }^{3} J=4.4 \mathrm{~Hz}, 2 \mathrm{H}, \mathrm{H}_{\beta}$-Zn-porph.), 8.75 (d, ${ }^{3} J=4.8 \mathrm{~Hz}, 2 \mathrm{H}, \mathrm{H}_{\beta}-\mathrm{Zn}$-porph.), 8.70 (s, $\left.1 \mathrm{H}, \mathrm{H}_{\text {pyrim. }}\right), 8.52\left(\mathrm{~d},{ }^{3} J=4.4 \mathrm{~Hz}, 2 \mathrm{H}, \mathrm{H}_{\beta}-\mathrm{Zn}\right.$-porph.), $8.11\left(\mathrm{~d},{ }^{3} J=8.4 \mathrm{~Hz}, 2 \mathrm{H}\right), 7.62\left(\mathrm{~d},{ }^{3} J=7.4 \mathrm{~Hz}\right.$, 2H, $\left.\mathrm{H}_{o}\right), 7.49\left(\mathrm{t},{ }^{3} J=7.4 \mathrm{~Hz}, 2 \mathrm{H}, \mathrm{H}_{m}\right), 7.41\left(\mathrm{t},{ }^{3} J=7.4 \mathrm{~Hz}, 1 \mathrm{H}, \mathrm{H}_{p}\right), 7.33\left(\mathrm{~d},{ }^{3} J=8.4 \mathrm{~Hz}, 2 \mathrm{H}\right), 7.28(\mathrm{~s}$, 4H, $\mathrm{H}_{\text {mesit. }}$ ), 5.35 (s, 2H, CH$\left.)_{2}\right), 2.63(\mathrm{~s}, 6 \mathrm{H}), 1.83(\mathrm{~s}, 12 \mathrm{H}) ;{ }^{13} \mathrm{C} \mathrm{NMR}\left(75 \mathrm{MHz}, \mathrm{CDCl}_{3}\right) \delta 163.7(\mathrm{C}-\mathrm{Cl})$, 158.6, $157.3\left(\mathrm{CH}_{\text {pyrim. }}\right), 150.5$ ( $\mathrm{C}_{\alpha}$-Zn-porph. $), 150.4\left(\mathrm{C}_{\alpha}-\mathrm{Zn}\right.$-porph. $), 149.8\left(\mathrm{C}_{\alpha}-\mathrm{Zn}\right.$-porph. $), 148.1\left(\mathrm{C}_{\alpha-}\right.$ Zn-porph.), 139.2, 138.6, 137.6, 137.0, $135.4(\mathrm{CH}-m-\mathrm{OBn}), 135.1,133.0(\mathrm{CH}), 132.4(\mathrm{CH}), 130.9$ $(\mathrm{CH}), 128.9(\mathrm{CH}), 128.7(\mathrm{CH}), 128.1(\mathrm{CH}), 127.7\left(\mathrm{CH}_{\text {mesit. }}\right)$, 122.0, 120.0, $113.0(\mathrm{CH}-o-\mathrm{OBn}), 107.4$ (meso-C), $70.4\left(\mathrm{CH}_{2}\right), 21.7\left(\mathrm{CH}_{3}\right), 21.5\left(\mathrm{CH}_{3}\right)$; UV-vis $\left(\mathrm{CH}_{2} \mathrm{Cl}_{2}\right) \lambda_{\max }(\log \varepsilon) 294.2(4.408), 402.0$ (4.671), 422.6 (5.759), 551.0 (4.374), 590.6 (3.559).

$\mathbf{G}_{\mathbf{1}}$-Dendron $\left(\mathbf{M}_{\mathbf{1}}=\mathbf{M}_{\mathbf{2}}=\mathbf{H}, \mathbf{H} ; \mathbf{R}=\mathbf{B n}\right)(\mathbf{5 a}) .{ }^{13} \mathrm{C} \mathrm{NMR}\left(75 \mathrm{MHz}, \mathrm{CDCl}_{3}\right) \delta 170.4\left(\mathrm{C}_{\text {pyrim. }}-\mathrm{OPh}\right)$, $158.8\left(\mathrm{CH}_{\text {pyrim. }}\right), 152.9$ ( $\mathrm{C}_{i}$-O-pyrim. $), 148.7\left(\mathrm{C}_{i}-\mathrm{t}\right.$-Bu $), 141.4,141.3,139.6,138.5,137.9,137.1,135.6$ $(\mathrm{CH}), 135.3(\mathrm{CH}), 134.7,131.3$ (br, $\mathrm{CH}_{\beta}$-porph.), $129.8(\mathrm{CH}), 129.7(\mathrm{CH}), 128.7(\mathrm{CH}), 128.2,127.9$ $(\mathrm{CH}), 127.8(\mathrm{CH}), 121.6,121.4,121.0(\mathrm{CH}), 120.5,119.8$ (CH-o-O-pyrim.), 118.7 (meso-C), 118.5 (meso-C), 113.2 (CH-o-OBn), 109.4 (meso-C), 105.3 (meso-C), $70.5\left(\mathrm{CH}_{2}\right), 35.1,31.8\left(\mathrm{CH}_{3}\right), 21.9$ $\left(\mathrm{CH}_{3}\right), 21.5\left(\mathrm{CH}_{3}\right)$.

$\mathbf{G}_{\mathbf{1}}$-Dendron ( $\left.\mathbf{M}_{\mathbf{1}}=\mathbf{P t}, \mathbf{M}_{\mathbf{2}}=\mathbf{H}, \mathbf{H} ; \mathbf{R}=\mathbf{B n}\right)(\mathbf{5 b})$. Eluent $\mathrm{CH}_{2} \mathrm{Cl}_{2}$-hexane 1-1; Yield $93 \%$ (110 mg); MS (ESI) calcd for $\mathrm{C}_{191} \mathrm{H}_{194} \mathrm{~N}_{14} \mathrm{O}_{3} \mathrm{Pt}_{2}: 3123.8$; found: $\mathrm{m} / z$ 3124.3; ${ }^{1} \mathrm{H}$ NMR (400 MHz, $\mathrm{CDCl}_{3}$ ) $\delta 9.30$ (d, ${ }^{3} J=4.8 \mathrm{~Hz}, 2 \mathrm{H}, \mathrm{H}_{\beta}$-porph.), 9.24 (s, $1 \mathrm{H}, \mathrm{H}_{\text {pyrim. }}$ ), 8.96 (d, ${ }^{3} J=4.8 \mathrm{~Hz}, 2 \mathrm{H}, \mathrm{H}_{\beta}$-porph.), 8.87 (d, ${ }^{3} J=$ 
$4.7 \mathrm{~Hz}, 2 \mathrm{H}, \mathrm{H}_{\beta}$-porph.), 8.78 (s, 8H, $\mathrm{H}_{\beta}$-Pt-porph.), 8.74 (d, ${ }^{3} J=4.8 \mathrm{~Hz}, 2 \mathrm{H}, \mathrm{H}_{\beta}$-porph.), $8.72\left(\mathrm{~d},{ }^{3} J=\right.$

$5.1 \mathrm{~Hz}, 4 \mathrm{H}, \mathrm{H}_{\beta}$-Pt-porph.), 8.66 (d, ${ }^{3} J=5.1 \mathrm{~Hz}, 4 \mathrm{H}, \mathrm{H}_{\beta}$-Pt-porph.), 8.17 (d, $\left.{ }^{3} J=8.5 \mathrm{~Hz}, 2 \mathrm{H}\right), 8.07$ (d, $\left.{ }^{3} J=8.5 \mathrm{~Hz}, 4 \mathrm{H}\right), 7.99\left(\mathrm{~d},{ }^{4} J=1.7 \mathrm{~Hz}, 4 \mathrm{H}\right), 7.98\left(\mathrm{~d},{ }^{4} J=1.7 \mathrm{~Hz}, 8 \mathrm{H}\right), 7.77-7.74(\mathrm{~m}, 6 \mathrm{H}), 7.62\left(\mathrm{~d},{ }^{3} J=\right.$ $\left.7.3 \mathrm{~Hz}, 2 \mathrm{H}, \mathrm{H}_{o}\right), 7.50-7.45(\mathrm{~m}, 6 \mathrm{H}), 7.41\left(\mathrm{~d},{ }^{3} J=7.3 \mathrm{~Hz}, 1 \mathrm{H}, \mathrm{H}_{p}\right), 7.37\left(\mathrm{~d},{ }^{3} J=8.6 \mathrm{~Hz}, 2 \mathrm{H}\right), 7.31(\mathrm{~s}$, 4H, $\left.\mathrm{H}_{\text {mesit. }}\right), 5.35\left(\mathrm{~s}, 2 \mathrm{H}, \mathrm{CH}_{2}\right), 2.62(\mathrm{~s}, 6 \mathrm{H}), 1.96(\mathrm{~s}, 12 \mathrm{H}), 1.49(\mathrm{~s}, 36 \mathrm{H}), 1.48(\mathrm{~s}, 72 \mathrm{H}),-2.25\left(\mathrm{~s}_{\mathrm{br}}, 2 \mathrm{H}\right.$, $\mathrm{NH}) ;{ }^{13} \mathrm{C} \mathrm{NMR}\left(100 \mathrm{MHz}, \mathrm{CDCl}_{3}\right) \delta 170.4\left(\mathrm{C}_{\text {pyrim. }}-\mathrm{OPh}\right), 158.8\left(\mathrm{C}_{i^{-}} \mathrm{OBn}\right), 158.2\left(\mathrm{CH}_{\text {pyrim. }}\right), 152.9\left(\mathrm{C}_{i^{-}}\right.$ O-pyrim.), 148.9 ( $\mathrm{C}_{i}-t$-Bu), 147-145 ( $\mathrm{C}_{\alpha}$-NH-porph.), 141.1 and 141.0 ( $\mathrm{C}_{\alpha}$-Pt-porph.), 140.7, 140.53, 140.49, 139.6, 138.7, 138.5, 137.8, $137.1\left(\mathrm{C}_{i, \mathrm{Bn}}\right), 135.6$ (CH-m-OBn), 134.7, 134.6 (CH-m-O-pyrim.), 132-128 ( $\mathrm{CH}_{\beta}$-NH-porph.), 130.97 ( $\mathrm{CH}_{\beta}-\mathrm{Pt}$-porph.), $130.89\left(\mathrm{CH}_{\beta}-\mathrm{Pt}\right.$-porph.), $130.82\left(\mathrm{CH}_{\beta}\right.$-Pt-porph.), 130.3 ( $\mathrm{CH}_{\beta}$-Pt-porph.), $129.0(\mathrm{CH}), 128.7\left(\mathrm{CH}_{m, \mathrm{Bn}}\right), 128.2\left(\mathrm{CH}_{p, \mathrm{Bn}}\right), 127.9\left(\mathrm{CH}_{\text {mesit. }}\right), 127.8\left(\mathrm{CH}_{o, \mathrm{Bn}}\right)$, 123.7 (meso-C), 123.6 (meso-C), 121.1 (CH), 120.8 (meso-C), 120.5, 119.9 (CH-o-O-pyrim.), 118.7 (meso- $\mathrm{C}_{\text {mesit. }}$ ), 113.2 (CH-o-OBn), 109.4 (meso-C), 105.2 (meso-C), $70.5\left(\mathrm{CH}_{2}\right), 35.1,31.7\left(\mathrm{CH}_{3}\right), 21.8$ $\left(\mathrm{CH}_{3}\right), 21.5\left(\mathrm{CH}_{3}\right) ; \mathrm{UV}$-vis $\left(\mathrm{CH}_{2} \mathrm{Cl}_{2}\right) \lambda_{\max }(\log \varepsilon) 292.0$ (4.720), 404.9 (5.868), 420.4 (5.788), 511.9 (4.925), 541.2 (4.180), 591.0 (3.825), 647.1 (3.577).

$\mathbf{G}_{1}$-Dendron $\left(\mathbf{M}_{1}=\mathbf{M}_{2}=\mathbf{H}, \mathbf{H} ; \mathbf{R}=\mathbf{H}\right)(\mathbf{6 a})$. Eluent $\mathrm{CH}_{2} \mathrm{Cl}_{2} ;$ Yield $84 \%$ (98 mg); MS (ESI) calcd for $\mathrm{C}_{184} \mathrm{H}_{192} \mathrm{~N}_{14} \mathrm{O}_{3}: 2647.6$; found: $m / z$ 2648.0 $[\mathrm{M}+\mathrm{H}]^{+}, 1323.8[\mathrm{M}+2 \mathrm{H}]^{2+}, 883.2[\mathrm{M}+3 \mathrm{H}]^{3+} ;{ }^{1} \mathrm{H} \mathrm{NMR}$ (300 MHz, $\left.\mathrm{CDCl}_{3}\right) \delta 9.35$ (d, ${ }^{3} J=4.7 \mathrm{~Hz}, 2 \mathrm{H}, \mathrm{H}_{\beta}$-porph.mon.), 9.29 (s, $1 \mathrm{H}, \mathrm{H}_{\text {pyrim. }}$ ), 8.99 (d, ${ }^{3} J=4.6$ $\mathrm{Hz}, 2 \mathrm{H}, \mathrm{H}_{\beta}$-porph.mon.), 8.90-8.72 (m, 20H, $\mathrm{H}_{\beta}$-porph.), 8.18 (d, $\left.{ }^{3} J=8.2 \mathrm{~Hz}, 4 \mathrm{H}\right), 8.10-8.03$ (m, 14H), 7.80-7.76 (m, 6H), $7.52\left(\mathrm{~d},{ }^{3} J=8.3 \mathrm{~Hz}, 4 \mathrm{H}\right), 7.31$ (s, 4H, $\left.\mathrm{H}_{\text {mesit. }}\right), 7.08\left(\mathrm{~d},{ }^{3} J=8.1 \mathrm{~Hz}, 2 \mathrm{H}\right), 2.62$ $(\mathrm{s}, 6 \mathrm{H}), 1.97(\mathrm{~s}, 12 \mathrm{H}), 1.52(\mathrm{~s}, 36 \mathrm{H}), 1.50(\mathrm{~s}, 72 \mathrm{H}),-2.26\left(\mathrm{~s}_{\mathrm{br}}, 2 \mathrm{H}, \mathrm{NH}\right),-2.72\left(\mathrm{~s}_{\mathrm{br}}, 4 \mathrm{H}, \mathrm{NH}\right) ;{ }^{13} \mathrm{C}$ NMR $\left(75 \mathrm{MHz}, \mathrm{CDCl}_{3}\right) \delta 170.4\left(\mathrm{C}_{\text {pyrim. }}-\mathrm{OPh}\right), 158.8\left(\mathrm{CH}_{\text {pyrim. }}\right), 155.5\left(\mathrm{C}_{i}-\mathrm{OH}\right), 152.9\left(\mathrm{C}_{i}-\mathrm{O}-\right.$ pyrim. $), 148.7$ (C ${ }_{i}-t$-Bu), 150-143 (br, $\mathrm{C}_{\alpha}$-porph.), 141.3, 141.2, 139.5 (C-p-O-pyrim.), 138.4, 137.8, 135.6 (CH-mOH), 135.3 (CH-m-O-pyrim.), 134.4 (C-p-OH), 131.3 (br, $\mathrm{CH}_{\beta}$-porph.), 129.8 (CH), 129.7 (CH), 127.9 $\left(\mathrm{CH}_{\text {mesit. }}\right.$ ), 121.6, 121.4, $121.0(\mathrm{CH}), 120.4,119.8$ (CH-o-O-pyrim.), 118.7 (meso-C), 118.5 (meso-C), 
113.7 (CH-o-OH), 109.4 (meso-C), 105.2 (meso-C), 35.0, $31.8\left(\mathrm{CH}_{3}\right), 21.9\left(\mathrm{CH}_{3}\right), 21.5\left(\mathrm{CH}_{3}\right)$; UV-vis $\left(\mathrm{CH}_{2} \mathrm{Cl}_{2}\right) \lambda_{\max }(\log \varepsilon) 421.2$ (6.091), 516.8 (4.755), 552.5 (4.459), 591.4 (4.237), 647.6 (4.175); GPC $\left(\mathrm{CHCl}_{3}\right) M_{\mathrm{w}}=2498, M_{\mathrm{n}}=2347, \mathrm{PDI}=1.064$.

$\mathbf{G}_{\mathbf{1}}$-Dendron $\left(\mathbf{M}_{1}=\mathbf{P t}, \mathbf{M}_{2}=\mathbf{H}, \mathbf{H} ; \mathbf{R}=\mathbf{H}\right)(\mathbf{6 b})$. Eluent $\mathrm{CH}_{2} \mathrm{Cl}_{2}$-hexane 1-1; Yield $83 \%$ (77 mg); MS (MALDI) calcd for $\mathrm{C}_{184} \mathrm{H}_{188} \mathrm{~N}_{14} \mathrm{O}_{3} \mathrm{Pt}_{2}$ : 3033.7; found: $m / z$ 3034.2; ${ }^{1} \mathrm{H}$ NMR $\left(300 \mathrm{MHz}, \mathrm{CDCl}_{3}\right) \delta 9.32$ (d, ${ }^{3} J=4.4 \mathrm{~Hz}, 2 \mathrm{H}, \mathrm{H}_{\beta}$-porph.), 9.26 (s, $1 \mathrm{H}, \mathrm{H}_{\text {pyrim. }}$ ), 8.97 (d, ${ }^{3} J=4.4 \mathrm{~Hz}, 2 \mathrm{H}, \mathrm{H}_{\beta}$-porph.), 8.87-8.64 (m, 20H, $\mathrm{H}_{\beta}$-porph.), 8.13-8.05 (m, 6H), 8.02-7.96 (m, 12H), 7.77-7.72 (m, 6H), $7.49\left(\mathrm{~d},{ }^{3} \mathrm{~J}=8.0 \mathrm{~Hz}\right.$, 4H), $7.30\left(\mathrm{~s}, 4 \mathrm{H}, \mathrm{H}_{\text {mesit. }}\right), 7.11\left(\mathrm{~d},{ }^{3} J=8.8 \mathrm{~Hz}, 2 \mathrm{H}\right), 2.61(\mathrm{~s}, 6 \mathrm{H}), 1.96(\mathrm{~s}, 12 \mathrm{H}), 1.49$ (s, 36H), 1.48 (s, 72H), -2.28 ( $\left.\mathrm{s}_{\mathrm{br}}, 2 \mathrm{H}, \mathrm{NH}\right) ;{ }^{13} \mathrm{C} \mathrm{NMR}\left(75 \mathrm{MHz}, \mathrm{CDCl}_{3}\right) \delta 170.4\left(\mathrm{C}_{\text {pyrim. }}-\mathrm{OPh}\right), 158.2\left(\mathrm{CH}_{\text {pyrim. }}\right), 155.5$ $\left(\mathrm{C}_{i}-\mathrm{OH}\right), 152.9\left(\mathrm{C}_{i}-\mathrm{O}\right.$-pyrim. $), 148.8\left(\mathrm{C}_{i}-t-\mathrm{Bu}\right), 141.1$ and $141.0\left(\mathrm{C}_{\alpha}-\mathrm{Pt}\right.$-porph. $), 140.6,140.50,140.45$, 139.5, 138.7, 138.4, 137.8, 135.7 (CH-m-OH), 134.6 (CH-m-O-pyrim.), 134.5, 131.0 ( $\mathrm{CH}_{\beta}$-Pt-porph.), 130.9 ( $\mathrm{CH}_{\beta}$-Pt-porph.), 130.8 ( $\mathrm{CH}_{\beta}$-Pt-porph.), 130.3 ( $\mathrm{CH}_{\beta}$-Pt-porph.), $129.0(\mathrm{CH}), 127.9$ (CH), 123.7 , 123.6, 121.1 (CH), 120.8, 120.4, 119.9 (CH-o-O-pyrim.), 118.7 (meso- $\mathrm{C}_{\text {mesit. }}$ ), 113.7 (CH-o-OH), 109.3 (meso-C), 105.1 (meso-C), 35.0, $31.7\left(\mathrm{CH}_{3}\right), 21.8\left(\mathrm{CH}_{3}\right), 21.5\left(\mathrm{CH}_{3}\right) ; \mathrm{UV}$-vis $\left(\mathrm{CH}_{2} \mathrm{Cl}_{2}\right) \lambda_{\max }(\log \varepsilon)$ 292.0 (4.746), 404.9 (5.889), 420.1 (5.812), 511.9 (4.945), 541.7 (4.204), 591.1 (3.847), 647.0 (3.601).

$\mathbf{G}_{\mathbf{1}}$-Dendron $\left(\mathbf{M}_{1}=\mathbf{P t}, \mathbf{M}_{2}=\mathbf{Z n} ; \mathbf{R}=\mathbf{H}\right)(\mathbf{6 c}) .{ }^{13} \mathrm{C} \mathrm{NMR}\left(75 \mathrm{MHz}, \mathrm{CDCl}_{3}\right) \delta 170.5\left(\mathrm{C}_{\text {pyrim. }}-\mathrm{OPh}\right)$, $158.0\left(\mathrm{CH}_{\text {pyrim. }}\right), 155.3\left(\mathrm{C}_{i}-\mathrm{OH}\right), 153.0$ ( $\mathrm{C}_{i}-\mathrm{O}$-pyrim. $), 150.41\left(\mathrm{C}_{\alpha}-\mathrm{Zn}\right.$-porph.), 150.35 ( $\mathrm{C}_{\alpha}-\mathrm{Zn}$-porph. $)$, 150.1 ( $\mathrm{C}_{\alpha}$-Zn-porph.), 150.0 ( $\mathrm{C}_{\alpha}$-Zn-porph.), $148.9\left(\mathrm{C}_{i}-t-\mathrm{Bu}\right), 141.1$ and $141.0\left(\mathrm{C}_{\alpha}\right.$-Pt-porph.), 140.6, 140.51, 140.47, 139.4, 139.1, 138.9, 137.6, 135.6 (CH-m-OH), 135.2, 134.7 (CH-m-O-pyrim.), 132.7 (CH), $132.3(\mathrm{CH}), 131.0$ ( $\mathrm{CH}_{\beta}$-porph.), 130.94 ( $\mathrm{CH}_{\beta}$-porph.), $130.87\left(\mathrm{CH}_{\beta}\right.$-porph.), $130.7\left(\mathrm{CH}_{\beta}-\right.$ porph.), 130.4 ( $\mathrm{CH}_{\beta}$-porph.), 130.3 ( $\mathrm{CH}_{\beta}$-porph.), $129.0(\mathrm{CH}), 127.8(\mathrm{CH}), 123.7,123.6,122.3,121.1$ $(\mathrm{CH}), 120.8,120.1(\mathrm{CH}), 119.7,113.5(\mathrm{CH}-o-\mathrm{OH}), 109.9$ (meso-C), 105.6 (meso-C), 35.0, $31.7\left(\mathrm{CH}_{3}\right)$, $21.9\left(\mathrm{CH}_{3}\right), 21.5\left(\mathrm{CH}_{3}\right)$. 
$\mathbf{G}_{2}$-Dendron $\left(\mathbf{M}_{1}=\mathbf{M}_{2}=\mathbf{M}_{3}=\mathbf{H}, \mathbf{H} ; \mathbf{R}=\mathbf{B n}\right)$ (7a). Purification by preparative TLC (silica, eluent $\mathrm{CH}_{2} \mathrm{Cl}_{2}$-hexane 2-1); Yield 55\% (26 mg); MS (MALDI, BMPM) calcd for $\mathrm{C}_{423} \mathrm{H}_{426} \mathrm{~N}_{34} \mathrm{O}_{7}$ : 6098.1; found: $m / z$ 6096.8; ${ }^{1} \mathrm{H}$ NMR $\left(300 \mathrm{MHz}, \mathrm{CDCl}_{3}\right) \delta 9.34\left(\mathrm{~d},{ }^{3} J=5.1 \mathrm{~Hz}, 2 \mathrm{H}, \mathrm{H}_{\beta}\right.$-porph.), $9.30\left(\mathrm{~d},{ }^{3} J=\right.$ $5.1 \mathrm{~Hz}, 4 \mathrm{H}, \mathrm{H}_{\beta}$-porph.), 9.28 (s, 1H, $\mathrm{H}_{\text {pyrim. }}$ ), 9.24 (s, 2H, $\mathrm{H}_{\text {pyrim. }}$ ), 9.00-8.72 (m, 42H, $\mathrm{H}_{\beta}$-porph.), 8.66 (d, ${ }^{3} J=5.1 \mathrm{~Hz}, 4 \mathrm{H}, \mathrm{H}_{\beta}$-porph.), 8.62 (d, ${ }^{3} J=5.1 \mathrm{~Hz}, 4 \mathrm{H}, \mathrm{H}_{\beta}$-porph.), $8.20\left(\mathrm{~d},{ }^{3} J=8.0 \mathrm{~Hz}, 2 \mathrm{H}\right), 8.13(\mathrm{~d}$, $\left.{ }^{3} J=8.0 \mathrm{~Hz}, 12 \mathrm{H}\right), 8.06-8.01(\mathrm{~m}, 24 \mathrm{H}), 7.78-7.74(\mathrm{~m}, 12 \mathrm{H}), 7.65\left(\mathrm{~d},{ }^{3} J=6.7 \mathrm{~Hz}, 2 \mathrm{H}, \mathrm{H}_{o}\right), 7.54-7.38$ (m, 17H), 7.33 (s, 4H, $\mathrm{H}_{\text {mesit. }}$ ), 7.28 (s, 8H, $\left.\mathrm{H}_{\text {mesit. }}\right), 5.39$ (s, 2H, $\left.\mathrm{CH}_{2}\right), 2.65$ (s, 6H), 2.60 (s, $\left.12 \mathrm{H}\right), 1.97$ $(\mathrm{s}, 12 \mathrm{H}), 1.91(\mathrm{~s}, 24 \mathrm{H}), 1.50(\mathrm{~s}, 72 \mathrm{H}), 1.49(\mathrm{~s}, 144 \mathrm{H}),-2.25\left(\mathrm{~s}_{\mathrm{br}}, 2 \mathrm{H}, \mathrm{NH}\right),-2.37\left(\mathrm{~s}_{\mathrm{br}}, 4 \mathrm{H}, \mathrm{NH}\right),-2.76(\mathrm{~s}-$ br, 8H, NH); ${ }^{13} \mathrm{C}$ NMR (100 MHz, $\left.\mathrm{CDCl}_{3}\right) \delta 170.3\left(\mathrm{C}_{\text {pyrim. }}-\mathrm{OPh}\right), 158.6\left(\mathrm{C}_{i}-\mathrm{OBn}\right), 158.2(\mathrm{CH}), 152.9$ (C ${ }_{i}$-O-pyrim.), 150-145 ( $\mathrm{C}_{\alpha}$-porph.), 148.7, 148.5, 141.3, 141.2, 139.5, 138.3, 137.8, $135.6(\mathrm{CH})$, $135.2(\mathrm{CH}), 132-130$ ( $\mathrm{CH}_{\beta}$-porph.), $129.74(\mathrm{CH}), 129.66(\mathrm{CH}), 128.7\left(\mathrm{CH}_{\mathrm{Bn}}\right), 128.2\left(\mathrm{CH}_{\mathrm{Bn}}\right), 127.9$ $(\mathrm{CH}), 127.8\left(\mathrm{CH}_{\mathrm{Bn}}\right), 121.5,121.4,121.0(\mathrm{CH}), 119.7(\mathrm{CH}), 119.6(\mathrm{CH}), 118.7,118.5,113.2(\mathrm{CH})$, 109.5 (meso-C), 105.5 (meso-C), $70.5\left(\mathrm{CH}_{2}\right), 35.0,31.7\left(\mathrm{CH}_{3}\right), 21.8\left(\mathrm{CH}_{3}\right), 21.4\left(\mathrm{CH}_{3}\right)$; UV-vis $\left(\mathrm{CH}_{2} \mathrm{Cl}_{2}\right) \lambda_{\max }(\log \varepsilon) 421.0$ (6.363), 516.9 (5.065), 552.0 (4.774), 591.7 (4.578), 647.5 (4.475); GPC $\left(\mathrm{CHCl}_{3}\right) M_{\mathrm{w}}=5661, M_{\mathrm{n}}=5479, \mathrm{PDI}=1.033$.

$\mathbf{G}_{2}$-Dendron $\left(\mathbf{M}_{1}=\mathbf{P t}, \mathbf{M}_{2}=\mathbf{Z n}, \mathbf{M}_{3}=\mathbf{H}, \mathbf{H} ; \mathbf{R}=\mathbf{B n}\right)(\mathbf{7 b}) .{ }^{13} \mathrm{C}$ NMR $\left(100 \mathrm{MHz}, \mathrm{CDCl}_{3}\right) \delta 170.43$ and $170.39\left(\mathrm{C}_{\text {pyrim. }}-\mathrm{OPh}\right), 158.8\left(\mathrm{C}_{i}-\mathrm{OBn}\right), 157.9,152.96$ and $152.86\left(\mathrm{C}_{i^{-}} \mathrm{O}\right.$-pyrim. $), 150.27\left(\mathrm{C}_{\alpha}-\mathrm{Zn}^{-}\right.$ porph.), 150.14 ( $\mathrm{C}_{\alpha}-\mathrm{Zn}$-porph.), 149.98 ( $\mathrm{C}_{\alpha}$-Zn-porph.), 149.95 ( $\mathrm{C}_{\alpha}-\mathrm{Zn}$-porph. $), 148.8\left(\mathrm{C}_{i}-t-\mathrm{Bu}\right)$, 141.05 ( $\mathrm{C}_{\alpha^{-}}$-Pt-porph.), 140.99 ( $\mathrm{C}_{\alpha}$-Pt-porph. $), 140.96$ ( $\mathrm{C}_{\alpha^{-}}$-Pt-porph.), 140.6 ( $\mathrm{C}_{\alpha^{-}}$-Pt-porph.), $140.5\left(\mathrm{C}_{\alpha^{-}}\right.$ Pt-porph.), 140.4 (C $\mathrm{C}_{\alpha}$-Pt-porph.), 139.6, 139.3, 139.0, 138.8, 138.5, 137.8, 137.5, $137.1\left(\mathrm{C}_{i, \mathrm{Bn}}\right), 135.6$ (CH-m-OBn), 135.1 (CH-m-O-pyrim.), 134.7, 134.6 (CH-m-O-pyrim.), 132.5 ( $\mathrm{CH}_{\beta}$-porph.), 132.2 ( $\mathrm{CH}_{\beta}$-porph.), 131.0 ( $\mathrm{CH}_{\beta}$-porph.), 130.92 ( $\mathrm{CH}_{\beta}$-porph.), 130.87 ( $\mathrm{CH}_{\beta}$-porph.), 130.78 ( $\mathrm{CH}_{\beta}$-porph.), 130.38 ( $\mathrm{CH}_{\beta}$-porph.), 130.35 ( $\mathrm{CH}_{\beta}$-porph.), 130.2 ( $\mathrm{CH}_{\beta}$-porph.), $129.0(\mathrm{CH}), 128.8\left(\mathrm{CH}_{\mathrm{Bn}}\right), 127.9$ $\left(\mathrm{CH}_{\mathrm{Bn}}\right), 127.8\left(\mathrm{CH}_{\text {mesit. }}\right), 127.7\left(\mathrm{CH}_{\mathrm{Bn}}\right), 123.6$ and 123.5 (meso-C), $121.1(\mathrm{CH}), 120.8$ and 120.3 (meso- 
C), 120.0 (СH-o-O-pyrim.), 119.7, 119.4 (CH-o-O-pyrim.), 118.7 (meso-C), 113.2 (CH-o-OBn), 109.9 (meso-C), 105.9 (meso-C), $70.5\left(\mathrm{CH}_{2}\right), 35.0,31.7\left(\mathrm{CH}_{3}\right), 21.84$ and $21.79\left(\mathrm{CH}_{3}\right), 21.48$ and 21.43 $\left(\mathrm{CH}_{3}\right)$.

$\mathbf{G}_{2}$-Dendron $\left(\mathbf{M}_{1}=\mathbf{P t}, \mathbf{M}_{2}=\mathbf{Z n}, \mathbf{M}_{3}=\mathbf{N i} ; \mathbf{R}=\mathbf{B n}\right)$ (7c). Metallation procedure: cfr. $4 \mathbf{c}(10 \mathrm{mg}$ scale). Purification by preparative TLC (silica, eluent $\mathrm{CH}_{2} \mathrm{Cl}_{2}$-petroleum ether 1-1); MS (MALDI) calcd for $\mathrm{C}_{423} \mathrm{H}_{412} \mathrm{~N}_{34} \mathrm{NiO}_{7} \mathrm{Pt}_{4} \mathrm{Zn}_{2}$ : 7053.8; found: $\mathrm{m} / z$ 7053.4; ${ }^{1} \mathrm{H}$ NMR (400 MHz, $\left.\mathrm{CDCl}_{3}\right) \delta 9.37$ (d, ${ }^{3} J=4.6$ Hz, 4H, H ${ }_{\beta}-\mathrm{Zn}$-porph.), 9.24 (d, ${ }^{3} J=5.0 \mathrm{~Hz}, 2 \mathrm{H}, \mathrm{H}_{\beta}$-Ni-porph.), 9.21 (s, 3H, $\mathrm{H}_{\text {pyrim. }}$ ), 9.02 (d, ${ }^{3} J=4.4$ $\mathrm{Hz}, 4 \mathrm{H}, \mathrm{H}_{\beta}-\mathrm{Zn}$-porph.), 8.89 (d, ${ }^{3} J=5.0 \mathrm{~Hz}, 2 \mathrm{H}, \mathrm{H}_{\beta}-\mathrm{Ni}$-porph.), 8.79 (d, ${ }^{3} J=4.7 \mathrm{~Hz}, 6 \mathrm{H}, \mathrm{H}_{\beta}-\mathrm{Zn} / \mathrm{Ni}-$ porph.), 8.76 (s, 16H, $\mathrm{H}_{\beta}$-Pt-porph.), 8.71 (d, ${ }^{3} J=4.6 \mathrm{~Hz}, 4 \mathrm{H}, \mathrm{H}_{\beta}-\mathrm{Zn}$-porph.), 8.68 (d, ${ }^{3} J=5.3 \mathrm{~Hz}, 8 \mathrm{H}$, $\mathrm{H}_{\beta}$-Pt-porph.), 8.65 (d, ${ }^{3} J=5.0 \mathrm{~Hz}, 2 \mathrm{H}, \mathrm{H}_{\beta}$-Ni-porph.), 8.62 (d, ${ }^{3} J=5.0 \mathrm{~Hz}, 8 \mathrm{H}, \mathrm{H}_{\beta}$-Pt-porph.), 8.15 (d, $\left.{ }^{3} J=8.1 \mathrm{~Hz}, 4 \mathrm{H}\right), 8.06\left(\mathrm{~d},{ }^{3} J=8.4 \mathrm{~Hz}, 8 \mathrm{H}\right), 8.02\left(\mathrm{~d},{ }^{3} J=8.4 \mathrm{~Hz}, 2 \mathrm{H}\right), 7.97\left(\mathrm{~d},{ }^{4} J=1.6 \mathrm{~Hz}, 8 \mathrm{H}\right), 7.95(\mathrm{~d}$, $\left.{ }^{4} J=1.6 \mathrm{~Hz}, 16 \mathrm{H}\right), 7.75(\mathrm{~s}, 4 \mathrm{H}), 7.73(\mathrm{~s}, 8 \mathrm{H}), 7.62\left(\mathrm{~d},{ }^{3} J=7.4 \mathrm{~Hz}, 2 \mathrm{H}, \mathrm{H}_{o}\right), 7.55-7.32(\mathrm{~m}, 21 \mathrm{H}), 7.28$ (s, 8H, $\mathrm{H}_{\text {mesit. }}$ ), 5.34 (s, 2H, $\left.\mathrm{CH}_{2}\right), 2.60$ (s, 12H), 2.58 (s, 6H), 1.93 (s, 12H), 1.89 (s, 24H), 1.48 (s, 72H), $1.46(\mathrm{~s}, 144 \mathrm{H}) ;{ }^{13} \mathrm{C}$ NMR (75 MHz, $\left.\mathrm{CDCl}_{3}\right) \delta 170.4\left(\mathrm{C}_{\text {pyrim. }}-\mathrm{OPh}\right), 150.3\left(\mathrm{C}_{\alpha}\right.$-Zn-porph. $), 150.2$

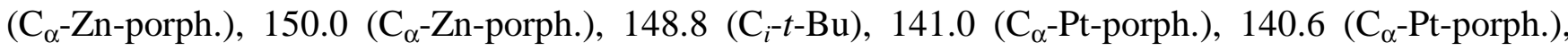
140.4 (C $\alpha_{\alpha}$-Pt-porph.), 139.3, 138.8, 134.6, 132.5, 130.8, 129.0, 128.7, 127.7, 123.5, 121.1, 120.0, 119.7, 35.0, $31.7\left(\mathrm{CH}_{3}\right), 21.8\left(\mathrm{CH}_{3}\right), 21.6\left(\mathrm{CH}_{3}\right), 21.4\left(\mathrm{CH}_{3}\right)$; UV-vis $\left(\mathrm{CH}_{2} \mathrm{Cl}_{2}\right) \lambda_{\max }(\log \varepsilon) 290.4$ (5.073), 405.4 (6.122), 424.2 (6.041), 511.3 (5.088), 543.6 (4.733), 588.4 (3.993).

\section{5-(4-Benzyloxyphenyl)-15-[4,6-bis(3,5-di-tert-butylphenoxy)pyrimidin-5-yl]-10,20-bis(2,4,6-tri-}

methylphenyl)porphyrin (8). Eluent $\mathrm{CH}_{2} \mathrm{Cl}_{2}$-hexane 2-1; Yield 80\% (102 mg); MS (ESI) calcd for $\mathrm{C}_{83} \mathrm{H}_{86} \mathrm{~N}_{6} \mathrm{O}_{3}$ : 1215.6; found: $m / z$ 1215.5; ${ }^{1} \mathrm{H}$ NMR (300 MHz, $\left.\mathrm{CDCl}_{3}\right) \delta 9.06\left(\mathrm{~d},{ }^{3} J=4.8 \mathrm{~Hz}, 2 \mathrm{H}, \mathrm{H}_{\beta^{-}}\right.$ porph.), 8.85 (s, 1H, $\mathrm{H}_{\text {pyrim. }}$ ), 8.79 (d, ${ }^{3} J=5.1 \mathrm{~Hz}, 4 \mathrm{H}, \mathrm{H}_{\beta}$-porph.), 8.64 (d, ${ }^{3} J=4.8 \mathrm{~Hz}, 2 \mathrm{H}, \mathrm{H}_{\beta}$-porph.), $8.11\left(\mathrm{~d},{ }^{3} J=8.4 \mathrm{~Hz}, 2 \mathrm{H}\right), 7.62\left(\mathrm{~d},{ }^{3} J=7.0 \mathrm{~Hz}, 2 \mathrm{H}, \mathrm{H}_{o}\right), 7.49\left(\mathrm{t},{ }^{3} J=7.0 \mathrm{~Hz}, 2 \mathrm{H}, \mathrm{H}_{m}\right), 7.41\left(\mathrm{~d},{ }^{3} J=7.0\right.$ 
$\left.\mathrm{Hz}, 1 \mathrm{H}, \mathrm{H}_{p}\right) 7.34\left(\mathrm{~d},{ }^{3} J=8.8 \mathrm{~Hz}, 2 \mathrm{H}\right), 7.29\left(\mathrm{~s}, 4 \mathrm{H}, \mathrm{H}_{\text {mesit. }}\right), 7.09\left(\mathrm{t},{ }^{4} J=1.5 \mathrm{~Hz}, 2 \mathrm{H}\right), 6.83\left(\mathrm{~d},{ }^{4} J=1.5\right.$

$\mathrm{Hz}, 4 \mathrm{H}), 5.34\left(\mathrm{~s}, 2 \mathrm{H}, \mathrm{CH}_{2}\right), 2.64(\mathrm{~s}, 6 \mathrm{H}), 1.85(\mathrm{~s}, 12 \mathrm{H}), 1.16(\mathrm{~s}, 36 \mathrm{H}),-2.46\left(\mathrm{~s}_{\mathrm{br}}, 2 \mathrm{H}, \mathrm{NH}\right)$.

\section{5-[4,6-Bis(3,5-di-tert-butylphenoxy)pyrimidin-5-yl]-15-(4-hydroxyphenyl)-10,20-bis(2,4,6-tri-}

methylphenyl)porphyrin (9). Eluent $\mathrm{CH}_{2} \mathrm{Cl}_{2}$; Yield $78 \%$ (76 mg); MS (ESI) calcd for $\mathrm{C}_{76} \mathrm{H}_{80} \mathrm{~N}_{6} \mathrm{O}_{3}$ :

1125.5; found: $m / z, 1125.5$; ${ }^{1} \mathrm{H}$ NMR $\left(300 \mathrm{MHz}, \mathrm{CDCl}_{3}\right) \delta 9.09\left(\mathrm{~d},{ }^{3} J=4.8 \mathrm{~Hz}, 2 \mathrm{H}, \mathrm{H}_{\beta}\right.$-porph.), 8.89 (s, $1 \mathrm{H}, \mathrm{H}_{\text {pyrim. }}$ ), 8.82 (d, ${ }^{3} J=4.8 \mathrm{~Hz}, 2 \mathrm{H}, \mathrm{H}_{\beta}$-porph.), 8.77 (d, ${ }^{3} J=4.8 \mathrm{~Hz}, 2 \mathrm{H}, \mathrm{H}_{\beta}$-porph.), 8.65 (d, ${ }^{3} J=4.8$

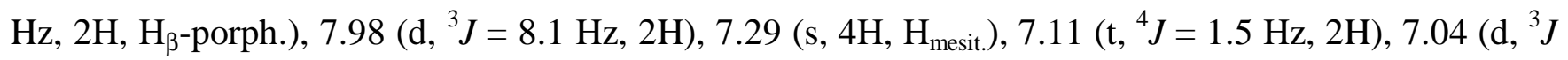
$=8.4 \mathrm{~Hz}, 2 \mathrm{H}), 6.85\left(\mathrm{~d},{ }^{4} \mathrm{~J}=1.5 \mathrm{~Hz}, 4 \mathrm{H}\right), 5.57\left(\mathrm{~s}_{\mathrm{br}}, 1 \mathrm{H}, \mathrm{OH}\right), 2.63(\mathrm{~s}, 6 \mathrm{H}), 1.86(\mathrm{~s}, 12 \mathrm{H}), 1.17(\mathrm{~s}, 36 \mathrm{H}),-$ $2.47\left(\mathrm{~s}_{\mathrm{br}}, 2 \mathrm{H}, \mathrm{NH}\right)$

G1-Dendron (10). Eluent $\mathrm{CH}_{2} \mathrm{Cl}_{2}$-hexane 5-1; Yield 73\% (20 mg); $\mathrm{MS}$ (ESI) calcd for $\mathrm{C}_{207} \mathrm{H}_{202} \mathrm{~N}_{18} \mathrm{O}_{7}: 3053.9$; found: $m / z$ 1528.2 $[\mathrm{M}+2 \mathrm{H}]^{2+}, 1125.8\left[\mathrm{G}_{1}\right] ;{ }^{1} \mathrm{H}$ NMR $\left(300 \mathrm{MHz}, \mathrm{CDCl}_{3}\right) \delta$ 9.28 (d, ${ }^{3} J=5.1 \mathrm{~Hz}, 2 \mathrm{H}, \mathrm{H}_{\beta}$-porph.), 9.23 (s, 1H, $\mathrm{H}_{\text {pyrim. }}$ ), 9.03 (d, ${ }^{3} J=5.1 \mathrm{~Hz}, 4 \mathrm{H}, \mathrm{H}_{\beta}-$ porph.), 8.94 (d, ${ }^{3} J=4.8 \mathrm{~Hz}, 2 \mathrm{H}, \mathrm{H}_{\beta}$-porph.), 8.83 (s, 2H, $\left.\mathrm{H}_{\text {pyrim }}\right), 8.86-8.50$ (m, 16H, $\mathrm{H}_{\beta}$-porph.), 8.18 (d, ${ }^{3} J=8.8 \mathrm{~Hz}$, $2 \mathrm{H}), 8.04\left(\mathrm{~d},{ }^{3} J=8.4 \mathrm{~Hz}, 4 \mathrm{H}\right), 7.66\left(\mathrm{~d},{ }^{3} J=7.3 \mathrm{~Hz}, 2 \mathrm{H}, \mathrm{H}_{o}\right), 7.52\left(\mathrm{t},{ }^{3} J=7.3 \mathrm{~Hz}, 2 \mathrm{H}, \mathrm{H}_{m}\right), 7.45-7.38$ (m, 7H), 7.29 (s, 4H, $\left.\mathrm{H}_{\text {mesit. }}\right), 7.25$ (s, 8H, $\left.\mathrm{H}_{\text {mesit. }}\right), 7.07\left(\mathrm{t},{ }^{4} \mathrm{~J}=1.5 \mathrm{~Hz}, 4 \mathrm{H}\right), 6.80\left(\mathrm{~d},{ }^{4} J=1.5 \mathrm{~Hz}, 8 \mathrm{H}\right)$, $5.39\left(\mathrm{~s}, 2 \mathrm{H}, \mathrm{CH}_{2}\right), 2.61(\mathrm{~s}, 18 \mathrm{H}), 1.92(\mathrm{~s}, 12 \mathrm{H}), 1.79(\mathrm{~s}, 24 \mathrm{H}), 1.14(\mathrm{~s}, 72 \mathrm{H}),-2.30\left(\mathrm{~s}_{\mathrm{br}}, 2 \mathrm{H}, \mathrm{NH}\right),-2.58$ $\left(\mathrm{s}_{\mathrm{br}}, 4 \mathrm{H}, \mathrm{NH}\right)$.

$\mathbf{G}_{\mathbf{0}}$-Dendrimer $\left(\mathbf{M}_{\mathbf{1}}=\mathbf{M}_{\mathbf{4}}=\mathbf{H}, \mathbf{H}\right)(\mathbf{1 1 a})$. MS (MALDI, IAA) $\mathrm{m} / z$ 4555.7; MS (MALDI, BMPM) $\mathrm{m} / z$ 4564.7; ${ }^{13} \mathrm{C}$ NMR (100 MHz, $\left.\mathrm{CDCl}_{3}\right) \delta 170.4\left(\mathrm{C}_{\text {pyrim. }}-\mathrm{OPh}\right), 158.2\left(\mathrm{CH}_{\text {pyrim }}\right), 152.9\left(\mathrm{C}_{i}\right.$-O-pyrim. $), 150-$ 145 (br, $\mathrm{C}_{\alpha}$-porph.), 148.7 ( $\mathrm{C}_{i}-\mathrm{t}$-Bu), 141.3, 141.2, 139.7, 139.5, 138.3, 138.0, 135.3 (CH-m-O-pyrim.), 131-130 ( $\mathrm{CH}_{\beta}$-porph.), $129.74(\mathrm{CH}), 129.65(\mathrm{CH}), 128.0\left(\mathrm{CH}_{\text {mesit. }}\right), 121.5(\mathrm{CH}), 121.4(\mathrm{CH}), 120.9$, 119.7 (СH-o-O-pyrim.), 119.0, 118.5 (meso-C), 109.3 (meso-C), 106.7 (meso-C), 35.01, 34.96, 31.72 $\left(\mathrm{CH}_{3}\right), 31.67\left(\mathrm{CH}_{3}\right), 22.0\left(\mathrm{CH}_{3}\right), 21.5\left(\mathrm{CH}_{3}\right)$.

$\mathbf{G}_{\mathbf{0}}$-Dendrimer $\left(\mathbf{M}_{1}=\mathbf{Z n}, \mathbf{M}_{4}=\mathbf{H}, \mathbf{H}\right)$ (11b). Eluent $\mathrm{CH}_{2} \mathrm{Cl}_{2}$; Yield 62\% (24 mg); MS (ESI) calcd for $\mathrm{C}_{318} \mathrm{H}_{334} \mathrm{~N}_{24} \mathrm{O}_{4} \mathrm{Zn}_{4}: 4817.8$; found: $m / z$. 2408.3 $[\mathrm{M}+2 \mathrm{H}]^{2+}, 1605.6[\mathrm{M}+3 \mathrm{H}]^{3+}, 1204.1[\mathrm{M}+4 \mathrm{H}]^{4+} ; \mathrm{MS}$ 
(MALDI, IAA) $\mathrm{m} / z$ 4804.7; ${ }^{1} \mathrm{H}$ NMR $\left(300 \mathrm{MHz}, \mathrm{CDCl}_{3}\right) \delta 9.31\left(\mathrm{~d},{ }^{3} J=4.8 \mathrm{~Hz}, 4 \mathrm{H}, \mathrm{H}_{\beta}\right.$-porph.core), 9.21 (s, 2H, $\mathrm{H}_{\text {pyrim. }}$ ), 8.97 (d, ${ }^{3} J=4.8 \mathrm{~Hz}, 4 \mathrm{H}, \mathrm{H}_{\beta}$-porph.core), 8.95-8.80 (m, 32H, $\mathrm{H}_{\beta}-\mathrm{Zn}$-porph.), 8.10 $\left(\mathrm{d},{ }^{3} \mathrm{~J}=8.5 \mathrm{~Hz}, 8 \mathrm{H}\right), 7.98\left(\mathrm{~d},{ }^{4} \mathrm{~J}=1.7 \mathrm{~Hz}, 8 \mathrm{H}\right), 7.95\left(\mathrm{~d},{ }^{4} \mathrm{~J}=1.7 \mathrm{~Hz}, 16 \mathrm{H}\right), 7.68\left(\mathrm{t},{ }^{4} \mathrm{~J}=1.7 \mathrm{~Hz}, 4 \mathrm{H}\right)$, $7.62\left(\mathrm{t},{ }^{4} \mathrm{~J}=1.7 \mathrm{~Hz}, 8 \mathrm{H}\right), 7.46\left(\mathrm{~d},{ }^{3} \mathrm{~J}=8.5 \mathrm{~Hz}, 8 \mathrm{H}\right), 7.28\left(\mathrm{~s}, 4 \mathrm{H}, \mathrm{H}_{\text {mesit. }}\right), 2.62$ (s, 6H), 2.09 (s, 12H), $1.42(\mathrm{~s}, 72 \mathrm{H}), 1.36(\mathrm{~s}, 144 \mathrm{H}),-2.08\left(\mathrm{~s}_{\mathrm{br}}, 2 \mathrm{H}, \mathrm{NH}\right) ; \mathrm{UV}$-vis $\left(\mathrm{CH}_{2} \mathrm{Cl}_{2}\right) \lambda_{\max }(\log \varepsilon) 422.7(6.372), 514.4$ (4.562), 549.5 (4.994), 588.8 (4.489), $647.1(3.625)$.

$\mathbf{G}_{\mathbf{1}}$-Dendrimer (12). Reaction procedure similar to $\mathrm{G}_{0}$-dendrimer 11a: reaction at $60{ }^{\circ} \mathrm{C}$ in $\mathrm{DMF}$ for 4 days with $\mathrm{K}_{2} \mathrm{CO}_{3}$ base and 4.4 equiv. $\mathrm{G}_{1}$-dendron 6a. Purification by preparative TLC (silica, eluent $\mathrm{CH}_{2} \mathrm{Cl}_{2}$-hexane 2-1); Yield 45\% (16 mg); MS (MALDI, BMPM) calcd for $\mathrm{C}_{782} \mathrm{H}_{798} \mathrm{~N}_{64} \mathrm{O}_{12}$ : 11285.1; found: $m / z 11284.8\left[\left(\mathrm{G}_{1}\right)_{4} \mathrm{P}\right], 8675.1\left[\left(\mathrm{G}_{1}\right)_{3} \mathrm{P}\right] ;{ }^{1} \mathrm{H}$ NMR $\left(300 \mathrm{MHz}, \mathrm{CDCl}_{3}\right) \delta 9.45-9.40\left(\mathrm{~m}, \mathrm{H}_{\beta^{-}}\right.$ porph.), 9.32-9.29 (m, $\mathrm{H}_{\beta}$-porph.), 9.23 (s, $\mathrm{H}_{\text {pyrim. }}$ ), 9.11-9.07 (m, $\mathrm{H}_{\beta}$-porph.), 8.97-8.92 (m, $\mathrm{H}_{\beta^{-}}$ porph.), 8.90-8.65 (m, $\mathrm{H}_{\beta}$-porph.), 8.24 (d, $\left.{ }^{3} J=8.0 \mathrm{~Hz}, \mathrm{G}_{1}\right), 8.13\left(\mathrm{~d},{ }^{3} J=8.0 \mathrm{~Hz}, \mathrm{G}_{0}\right), 8.03\left(\mathrm{~d},{ }^{4} J=1.7\right.$ $\mathrm{Hz}), 8.02\left(\mathrm{~d},{ }^{4} J=1.7 \mathrm{~Hz}\right), 7.76\left(\mathrm{~s}_{\mathrm{br}}\right), 7.74\left(\mathrm{~s}_{\mathrm{br}}\right), 7.59\left(\mathrm{~d},{ }^{3} J=8.4 \mathrm{~Hz}, \mathrm{G}_{1}\right), 7.47\left(\mathrm{~d},{ }^{3} J=8.4 \mathrm{~Hz}, \mathrm{G}_{0}\right), 7.26$ (s, $\left.\mathrm{H}_{\text {mesit. }}\right), 2.55(\mathrm{~s}), 1.93(\mathrm{~s}), 1.49(\mathrm{~s}), 1.47(\mathrm{~s}),-1.97\left(\mathrm{~s}_{\mathrm{br}}, \mathrm{NH}\right),-2.10\left(\mathrm{~s}_{\mathrm{br}}, \mathrm{NH}\right),-2.33\left(\mathrm{~s}_{\mathrm{br}}, \mathrm{NH}, \mathrm{G}_{1}\right),-2.77$ $\left(\mathrm{s}_{\mathrm{br}}, \mathrm{NH}, \mathrm{G}_{0}\right) ; \mathrm{GPC}\left(\mathrm{CHCl}_{3}\right) M_{\mathrm{w}}=8236, M_{\mathrm{n}}=7798, \mathrm{PDI}=1.056$. 
t28t $z^{-}$

$6058^{\circ} \mathrm{I}$

$2819^{\circ} \mathrm{Z}$

88EE $S$
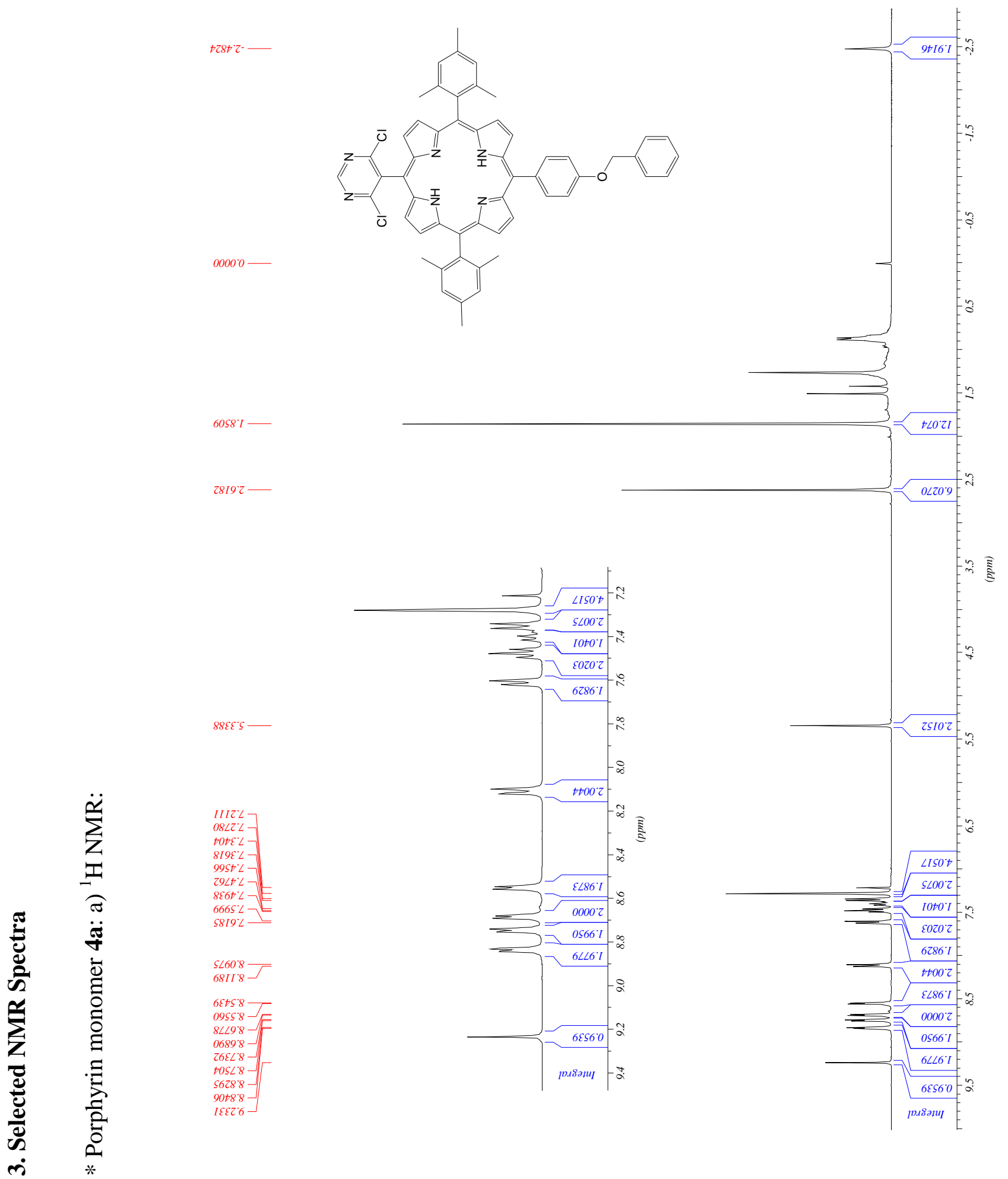
I8tt'IZ $95 \angle 9 \cdot I Z$

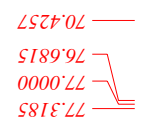

S8E8.90I

$I^{\prime} 80{ }^{\circ} \mathcal{E} I I$
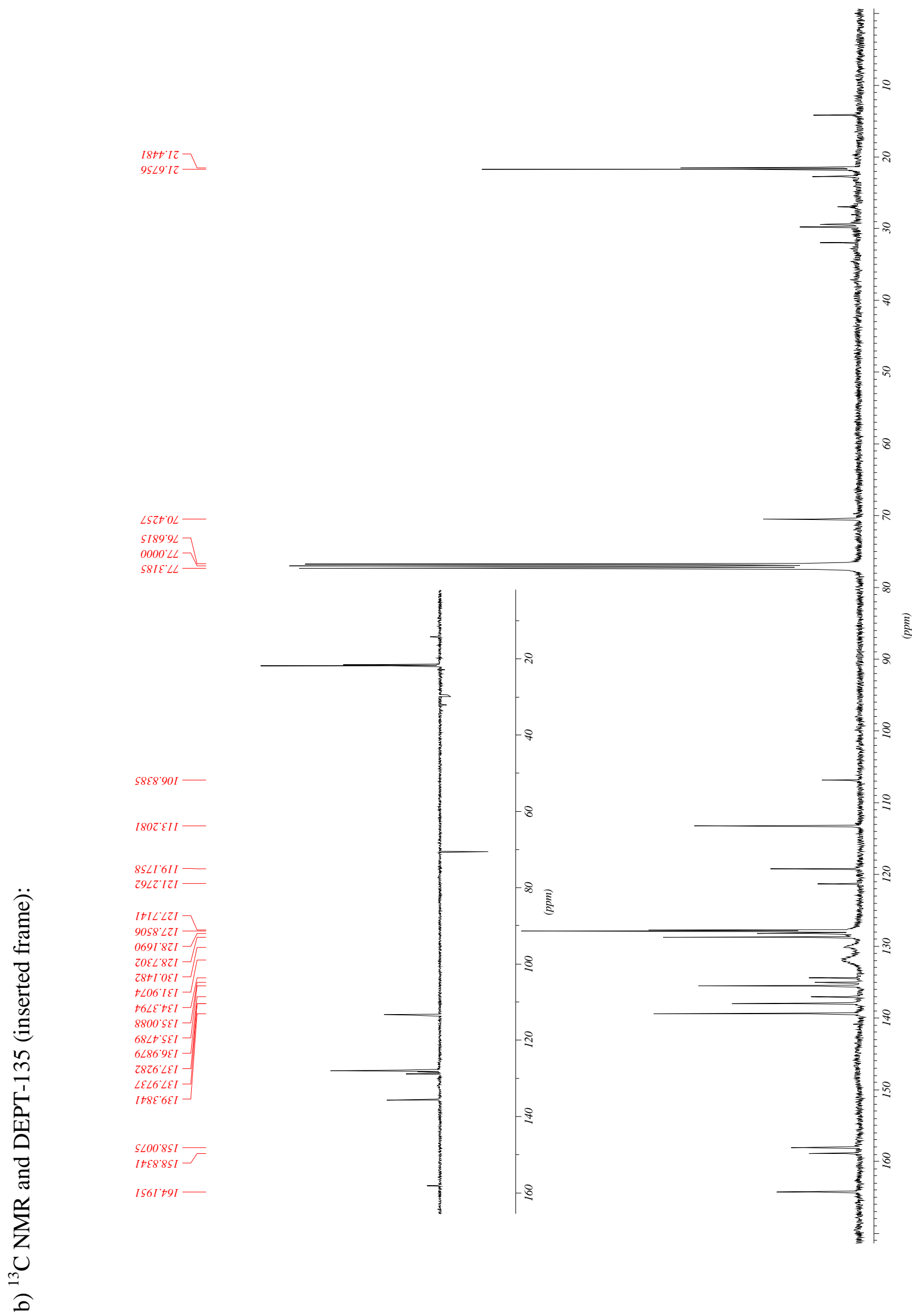


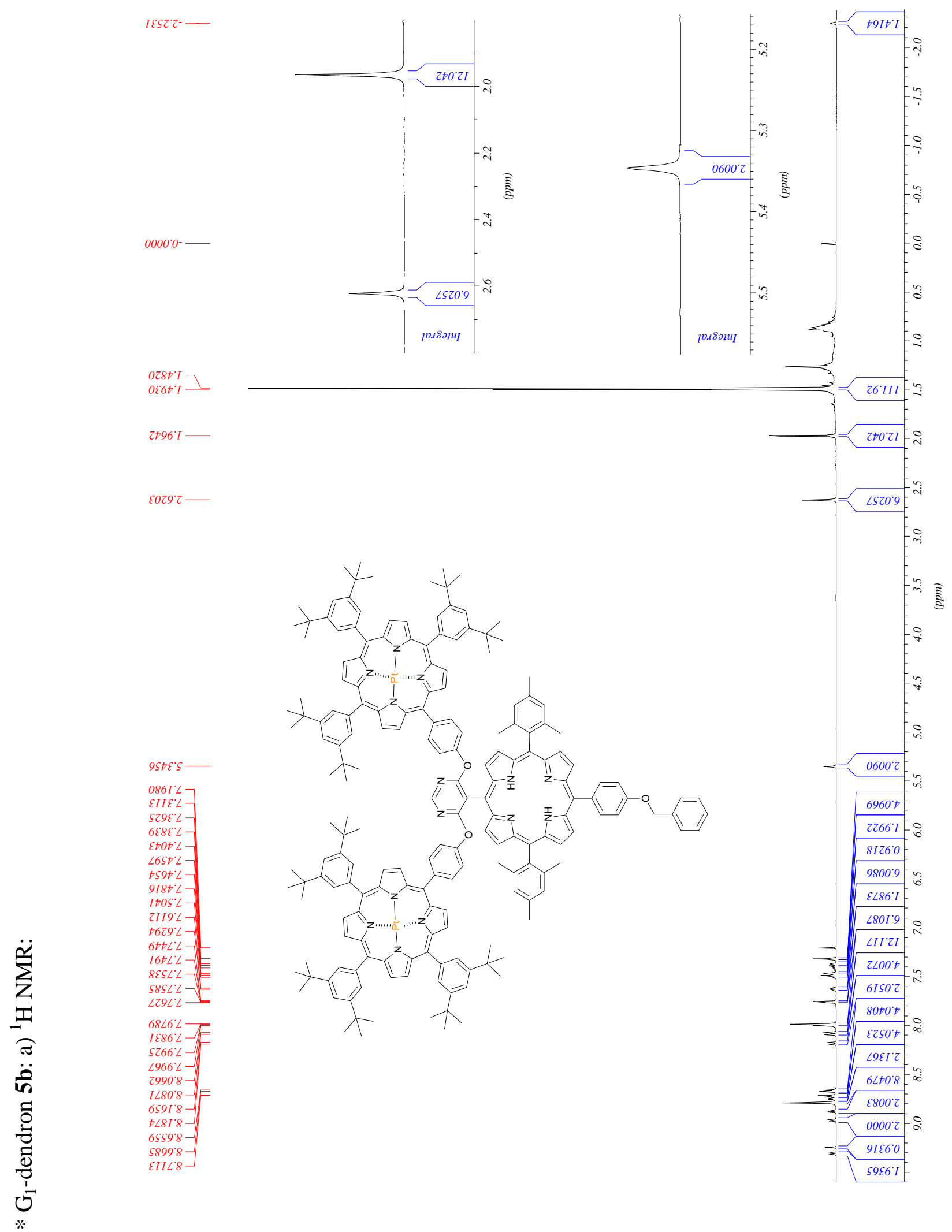




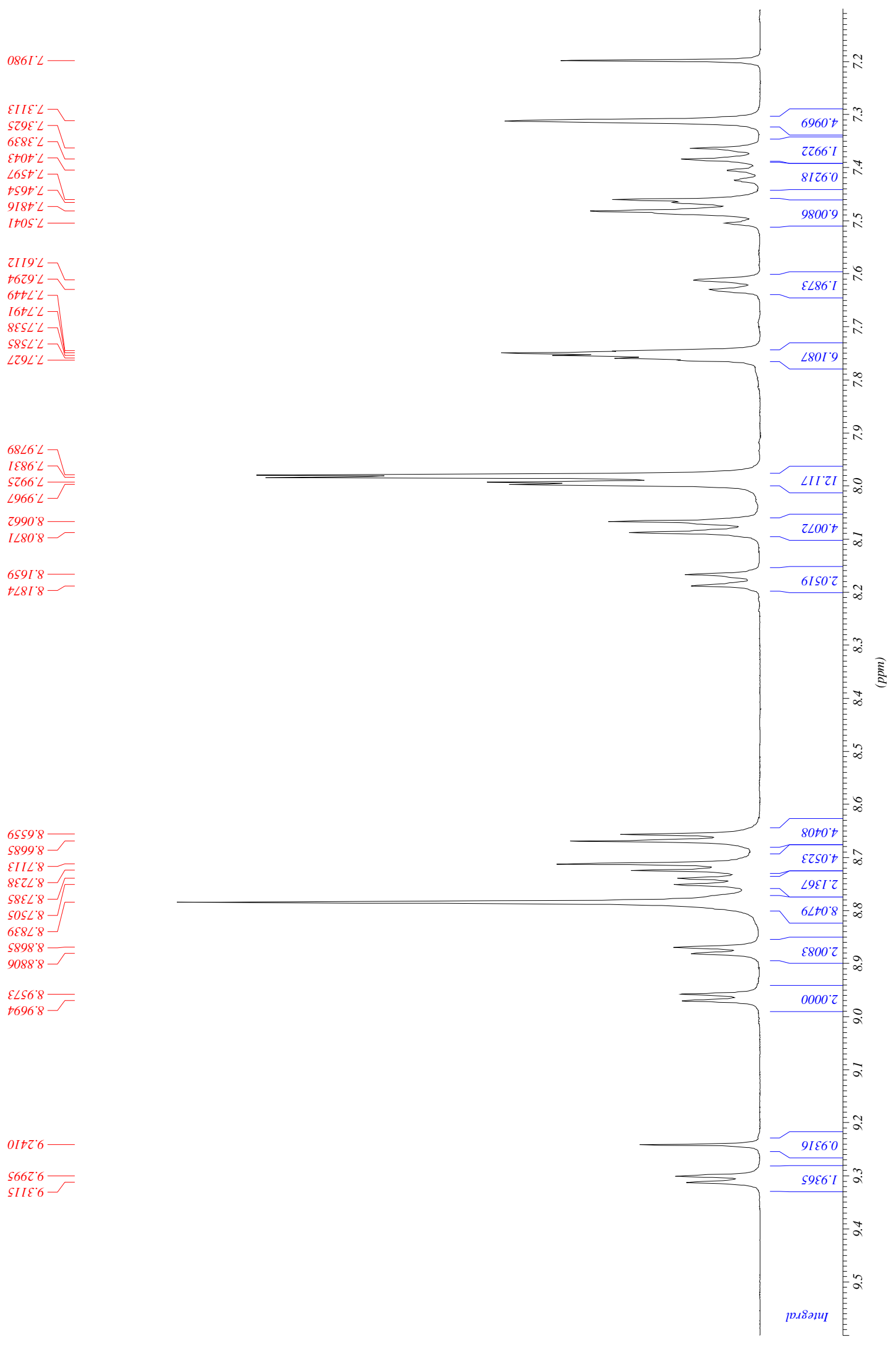

$68 \angle 6^{\circ} \mathrm{L}$

IE86 L

$\varsigma 266^{\circ} \mathrm{L}$

$\angle 966^{\circ} \mathrm{L}-$

$29908^{\circ}$

I $\angle 80^{\circ} 8-$

$65918-$

$+\angle 8 I^{\circ} 8-$

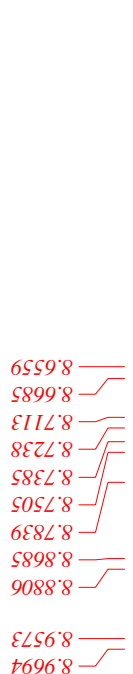


$098+12$

$t 2+8.12 \longrightarrow$

$08 \mathcal{L} L^{\circ} I \mathcal{L}-$

OSSt $04-$

SI89.9L

$0000^{\circ} \angle L$

$601 \varepsilon \angle L$

$9002 \cdot 5 O I-$

ZILE' $60 I-$
$\angle S I Z^{\circ} \mathcal{E} I I$

2099:8II

$8598^{\circ} 6 I I-$

$8828^{\circ} 02 \mathrm{I}$

OLII IZI

$\angle 8 S S^{\circ} \mathcal{E Z I}$

$\angle 6+9^{\circ} \varepsilon z I-$

$I \angle 9 L{ }^{\circ} \angle Z I-$

$6088^{\circ} \angle Z I$

$99 \angle I \cdot 8 Z I-$

ESt $\angle$ '8ZI
†086

zZ6z०OEI -

OEZ8.0EI -

$6868^{\circ} 0 \mathcal{E} I-$

$\angle \nrightarrow \angle 6 \circ 0 \mathcal{\circ} I$

$6909^{\circ}+\mathcal{E} I$

$O \mathcal{E} I \angle{ }^{\circ} \mathrm{E} I$

$8 \angle 09^{\circ} S \mathcal{}$ I

$9 I 0 I^{\circ} \angle E I$

$\angle t+8^{\circ} \angle E I-$

tIST $8 E I$

9I0L'8EI

6OSS $6 \mathcal{6 E I}$

2I6t'0tI

$\angle 9 E S^{\circ} 0 t I$

$9 S 99^{\circ} 0 t I$

8900 It I

S620'ItI -

2060'ItI

$z Z+6 \cdot z S I$

988L'8SI

$9 \angle 9 \mathcal{E}^{\circ} 0 \angle I-$

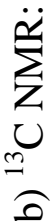

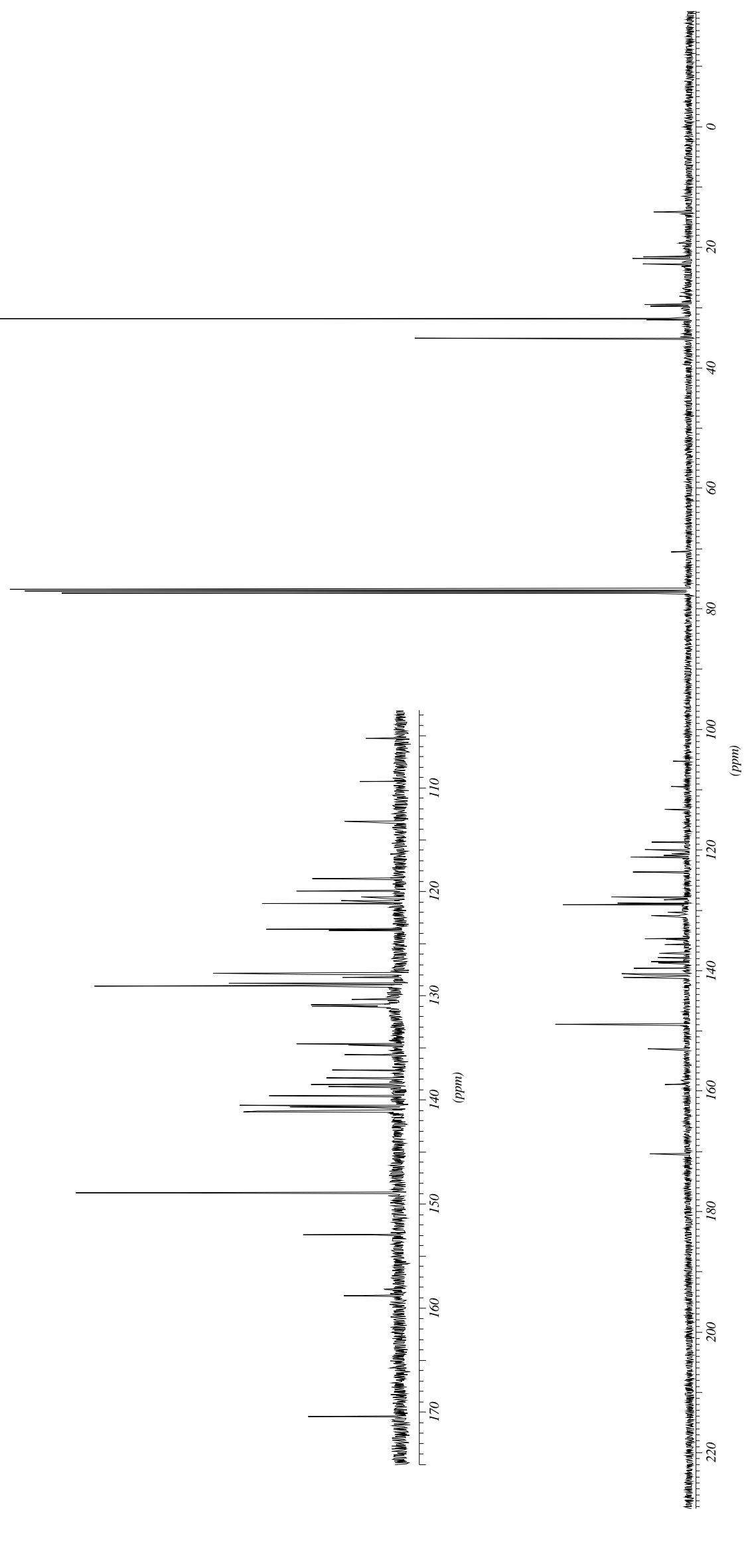


$\frac{n}{n}$

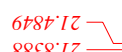

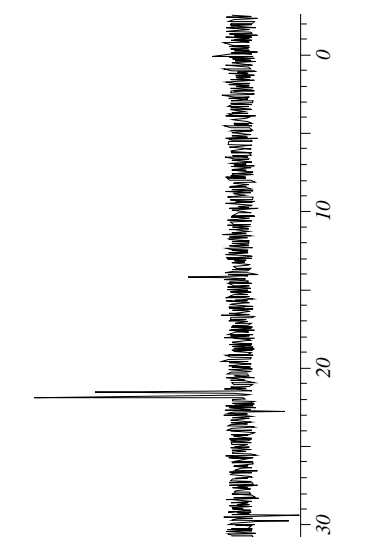

$9 z \varepsilon<1$

$\angle S S T^{\circ} O L$

LSIZ'EII-

$\ddot{n}$
$\frac{1}{1}$
$\frac{1}{0}$
0
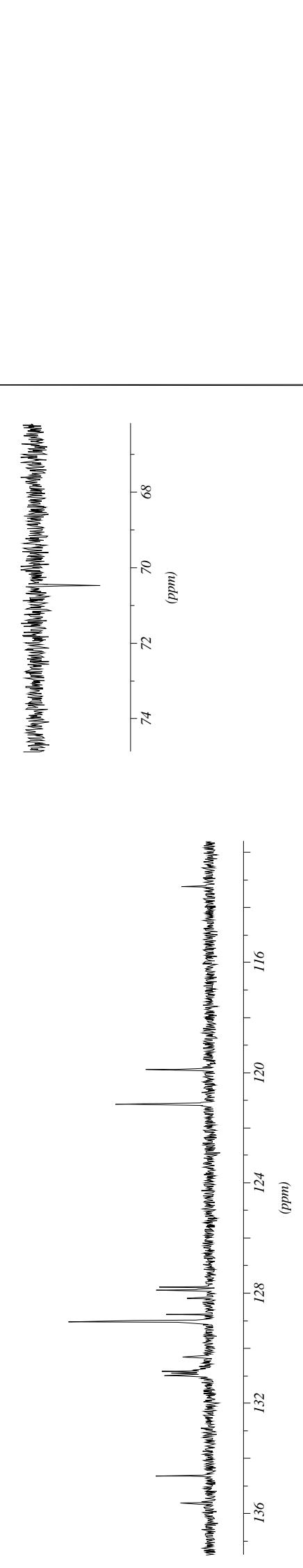

$6998.611-$

E9II IZI -

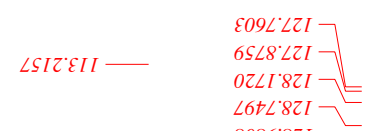

घ9II.IZI — EIEO $62 I$

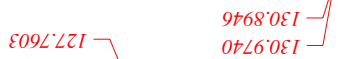

$6 S \angle 8 \cdot \angle Z I-\frac{}{\sqrt{F}}$
$0 Z \angle I \cdot 8 Z I-\sqrt{-}$

$\angle 6+\angle 8 Z I-\quad s 909^{\circ}+\varepsilon I$

8086821

IEO ${ }^{\circ} S \mathcal{E L}$

$\begin{array}{ll}1 & \text { trOE.OEI } \\ \text { ISIROEI }\end{array}$

$\angle S S+O O L$ 

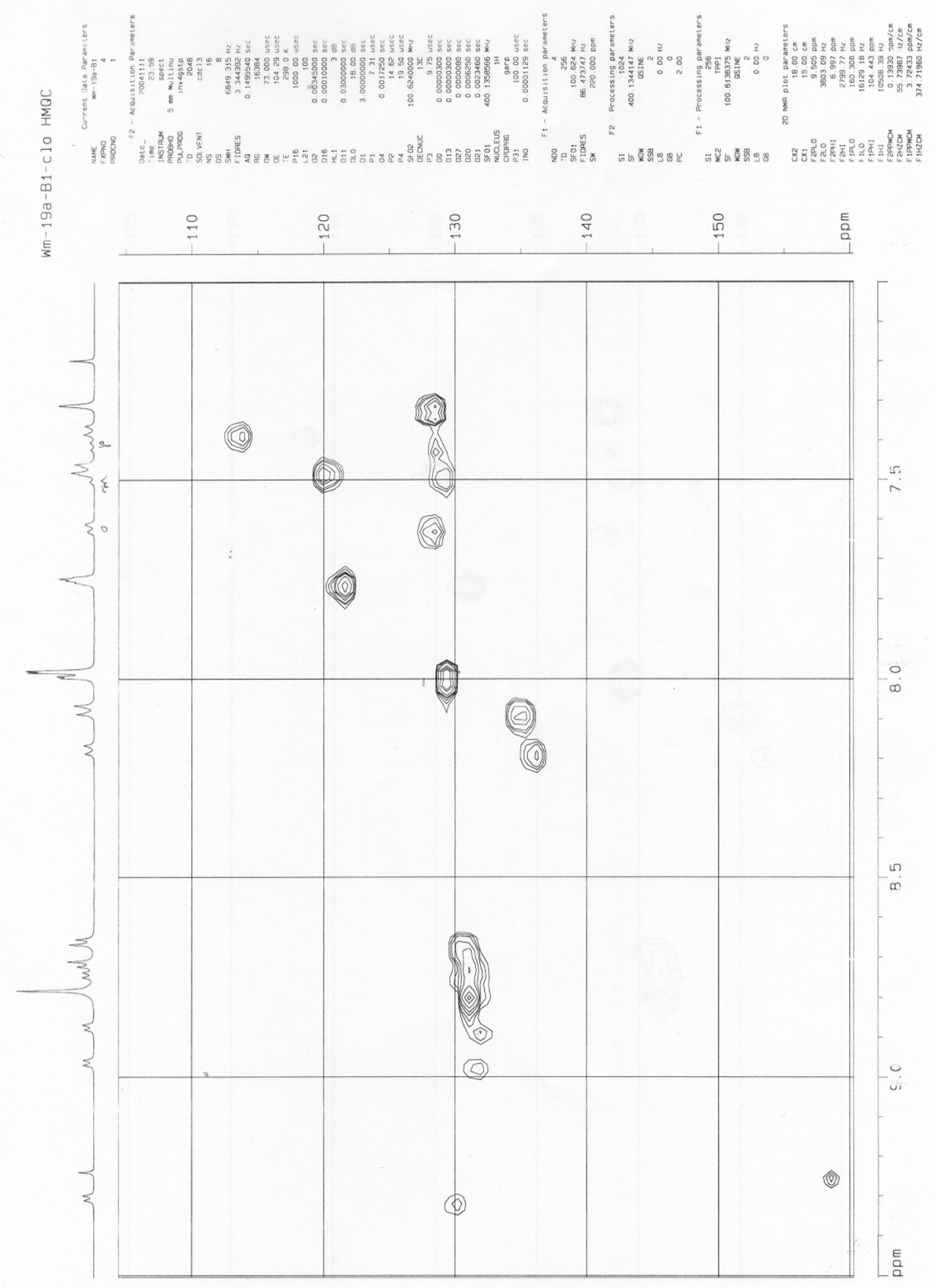

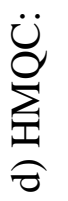


s

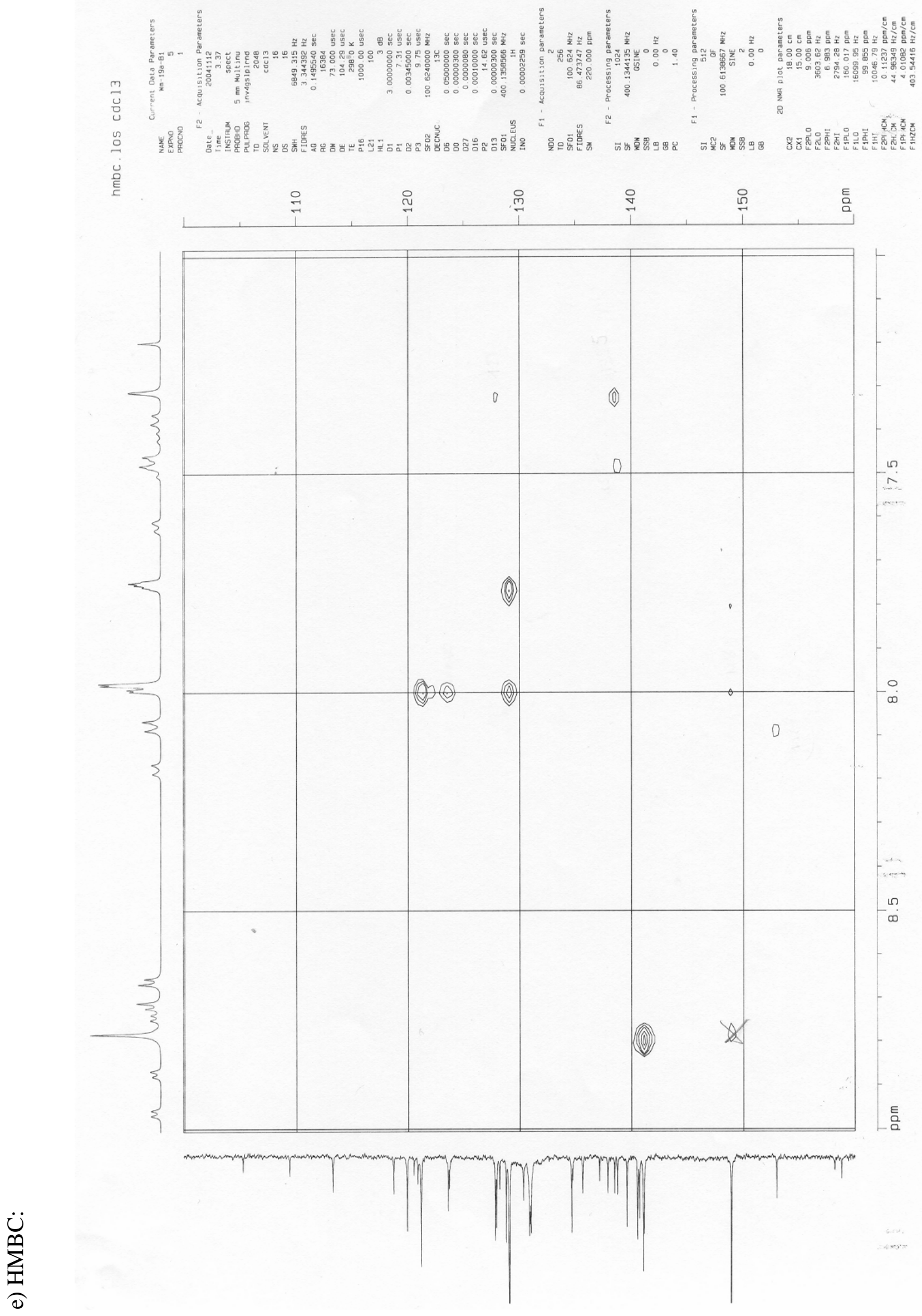


$\operatorname{oszz} z-$

$00000^{-}$
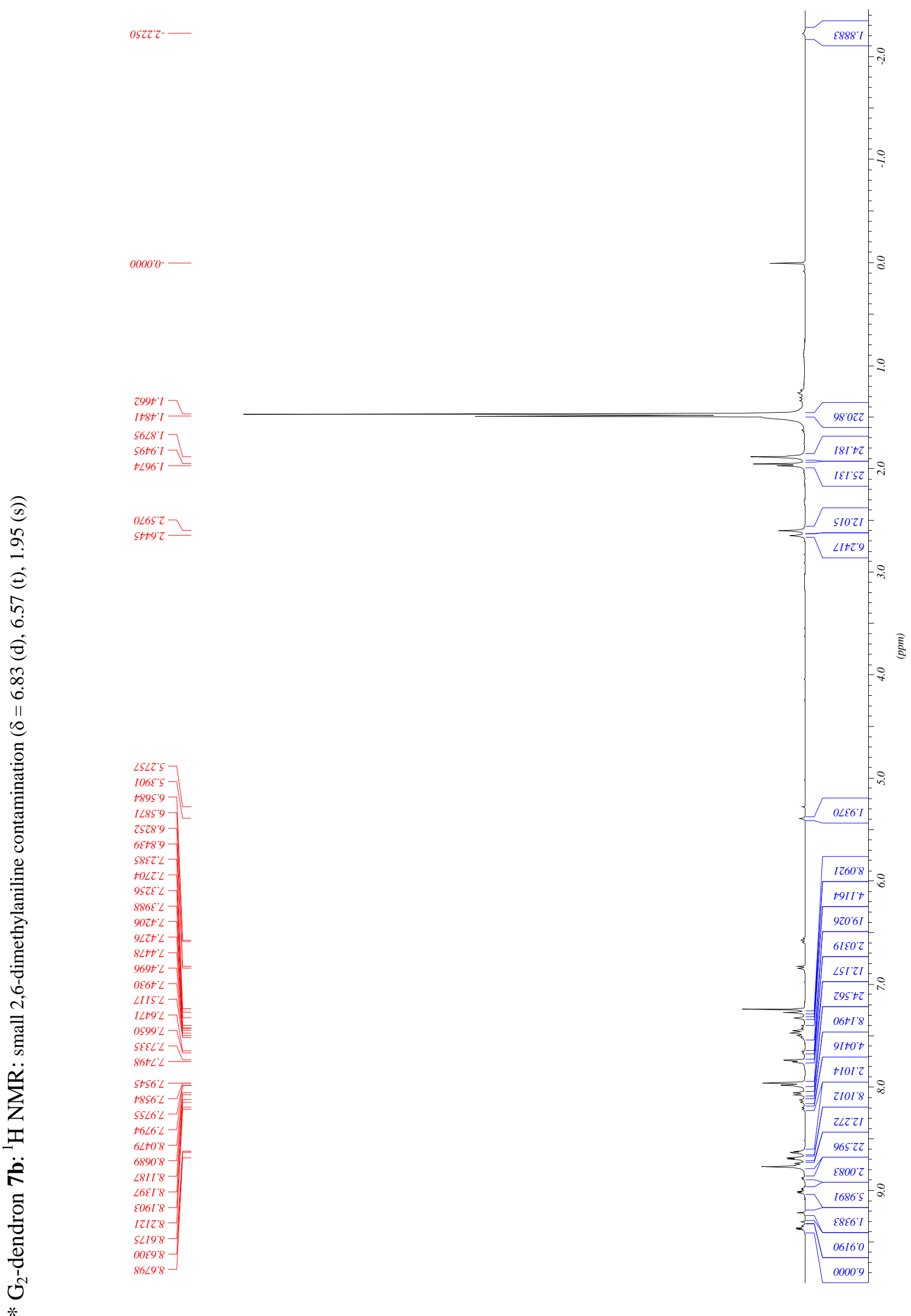

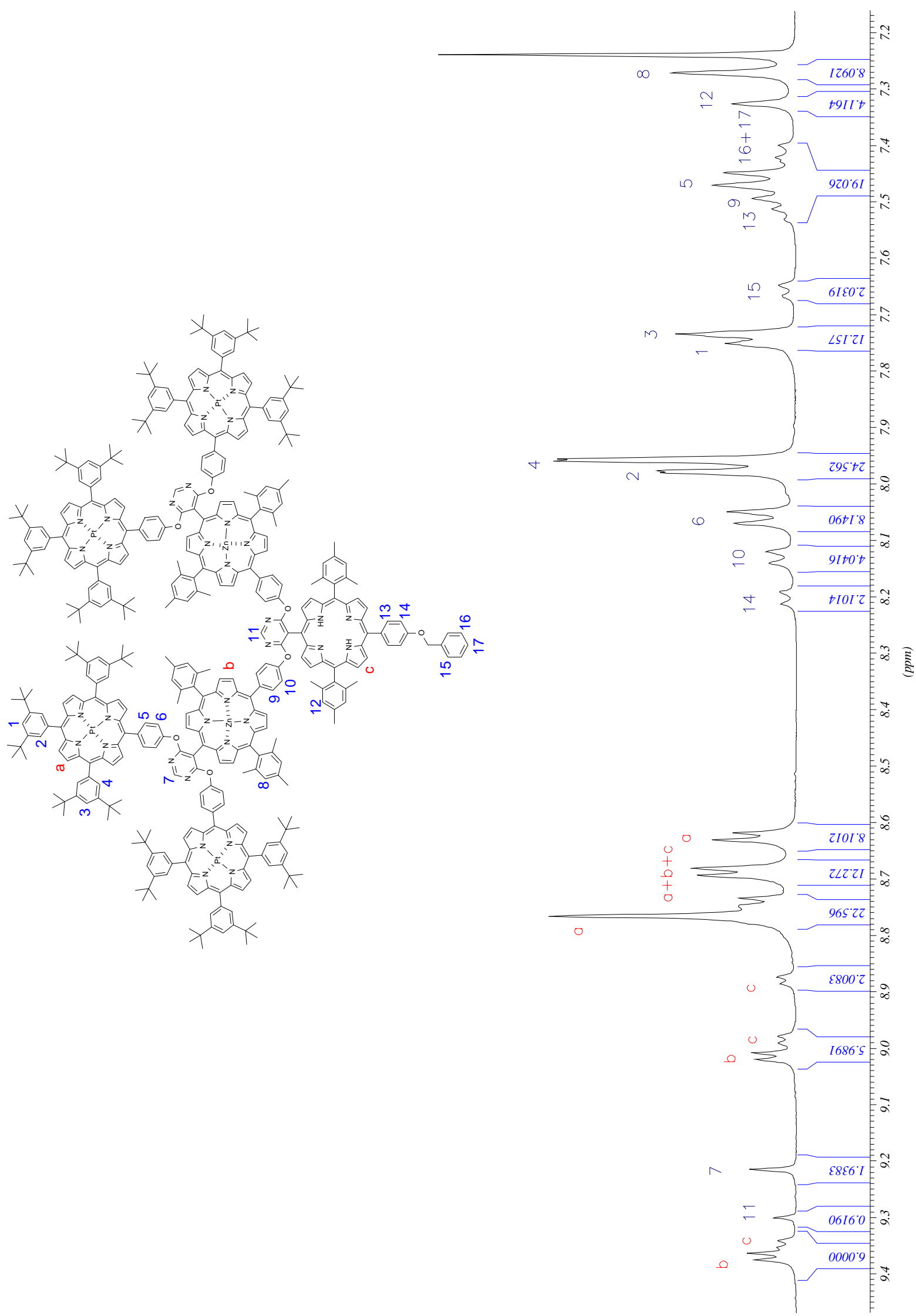
IE9t' I

$818+1$

$\angle 268^{\circ} I-$

$+2 \varepsilon 6{ }^{\circ} I$

z28s' 2

szo9' 2

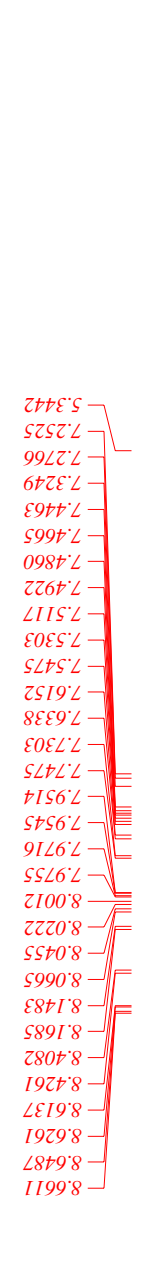

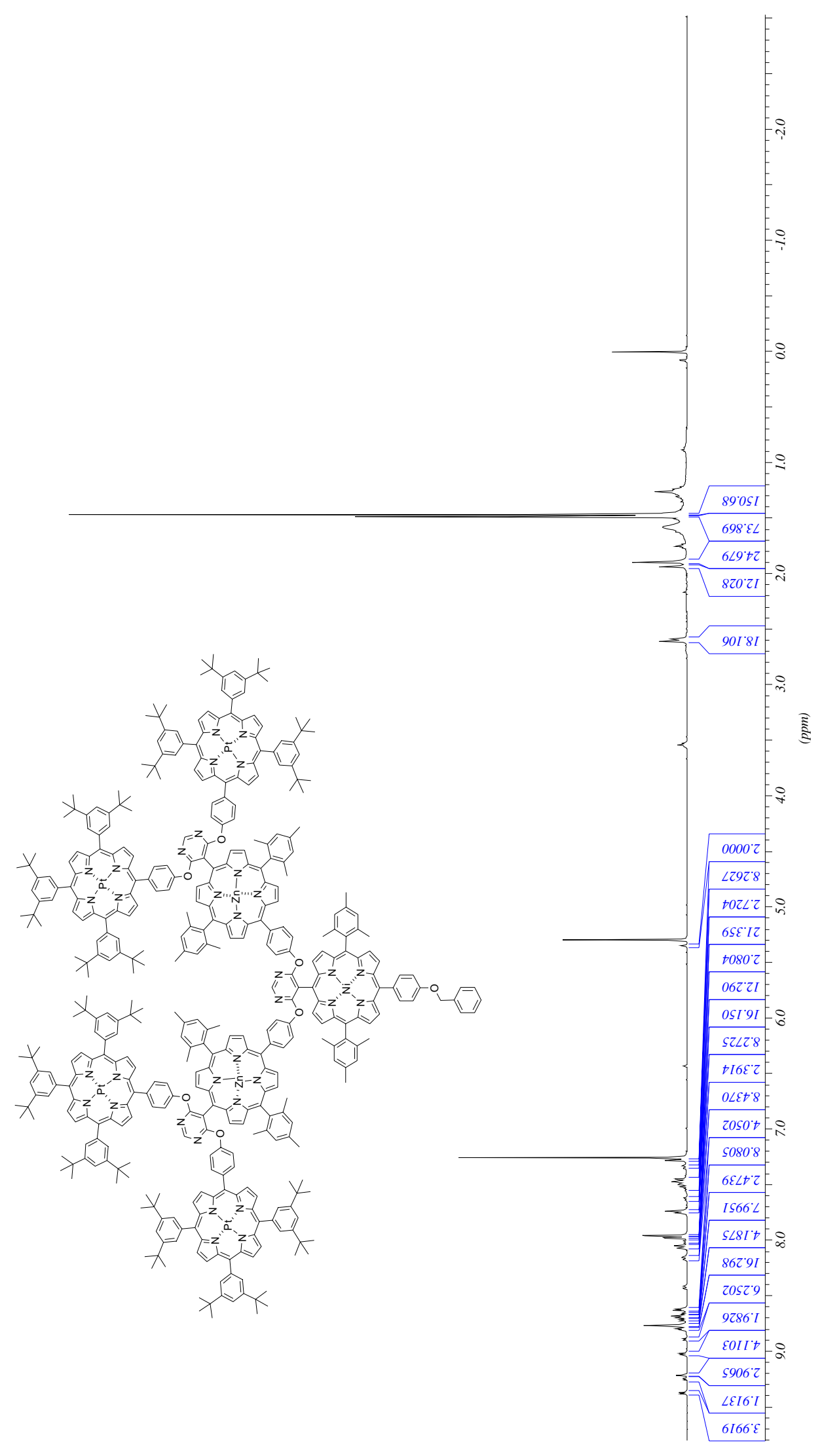




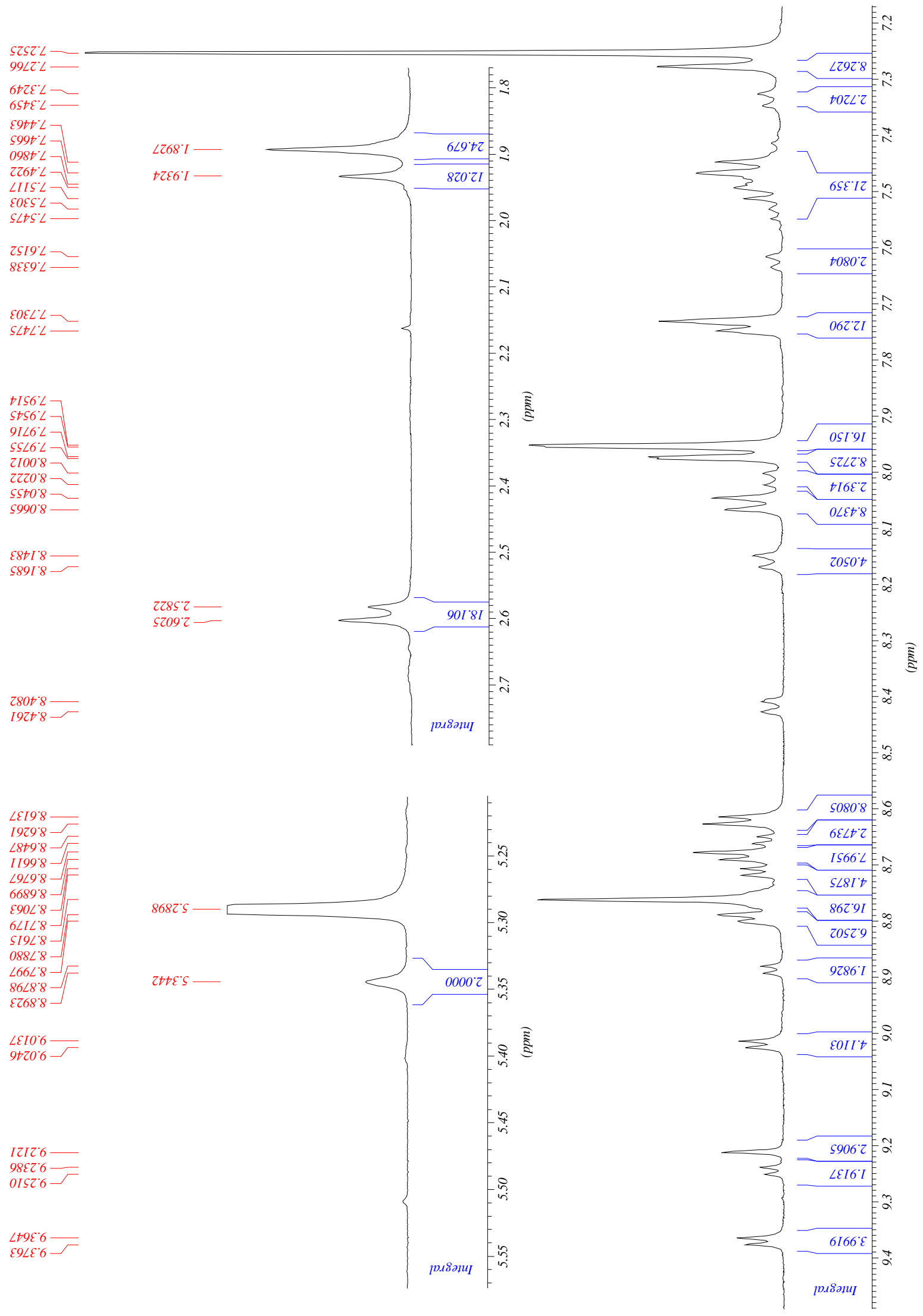


ฮี

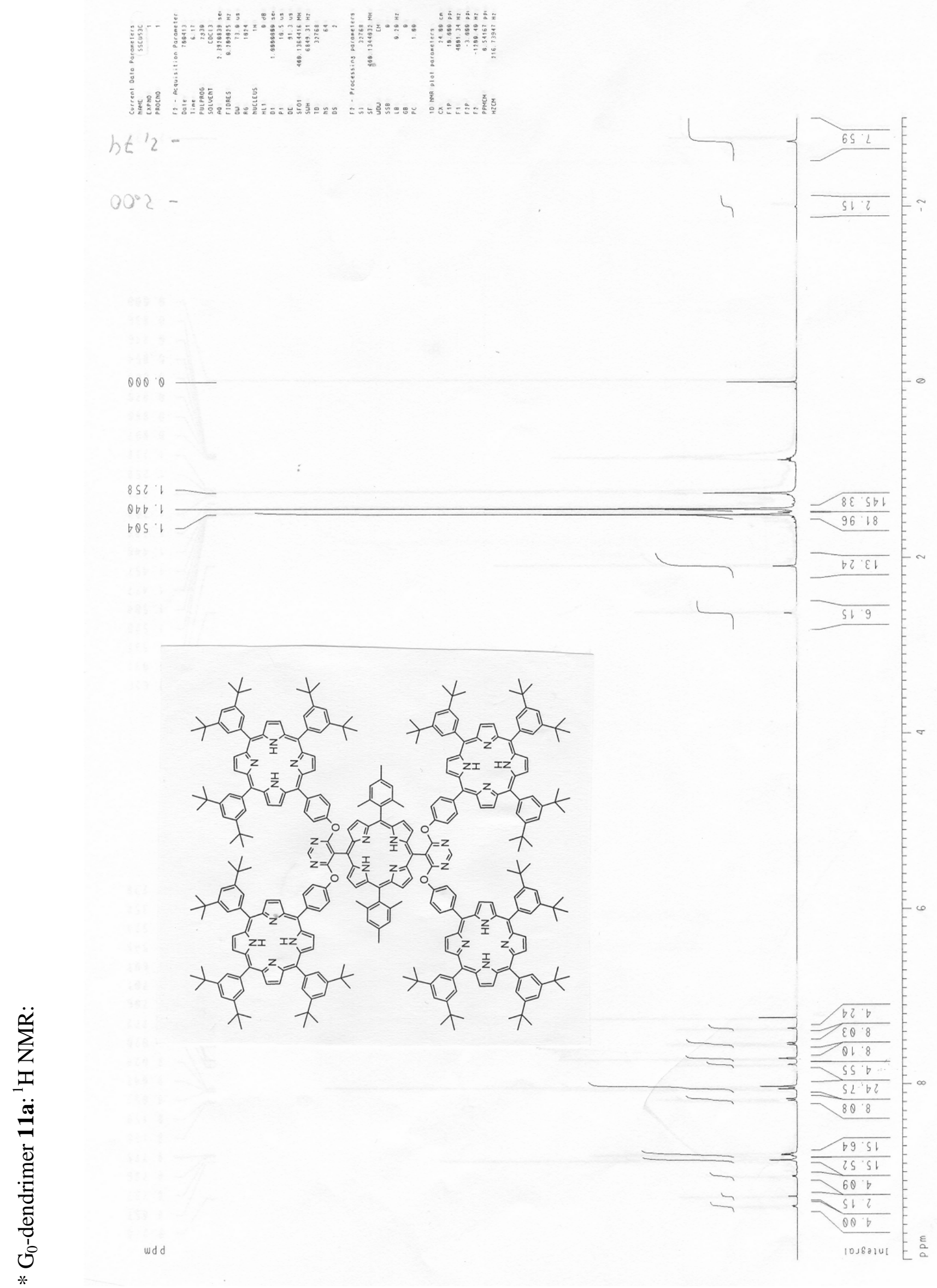




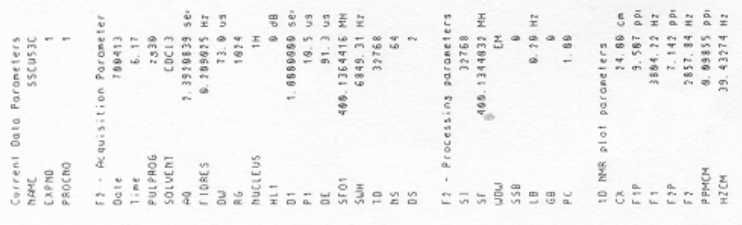

Q 8\& $2 L$

S\&SE $\mathrm{L}$

6EZS L

ZSBS $\mathrm{LL}$

$2 \angle 69^{\circ}$

SLOL

$\angle L 9 L L \longrightarrow$

$612 L$
$19 L 2 L$

5610.8
$8 \varepsilon 20.8$

$S \angle D Q \cdot 8-$

$8150 \cdot 8$

2651.8-

$6081.8-$

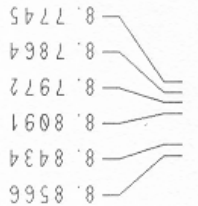

$b S E \theta 6 \longrightarrow$

$9 \angle 606 \div$

$20<26$

$\angle 9 L E \cdot 6$
$\angle 88 E \cdot 6$
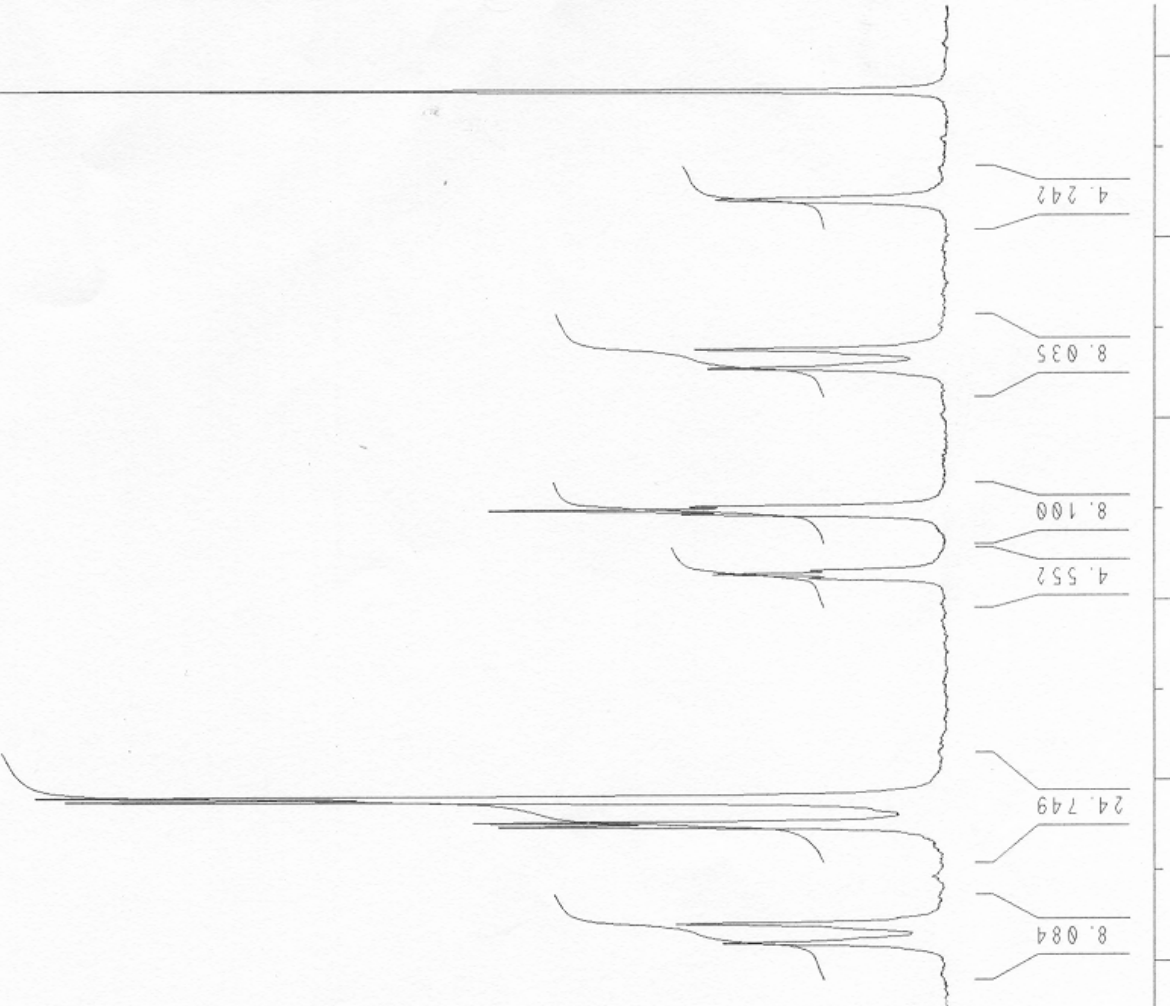

$+$

\section{$\infty$}

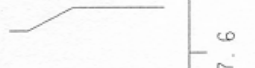

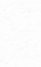
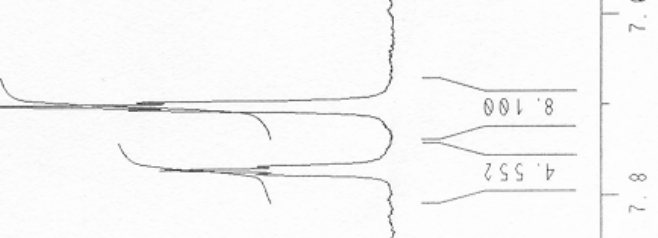


\section{Selected MS Spectra}

* $\mathrm{G}_{1}$-dendron $\mathbf{5 b}(\mathrm{ESI})$ :

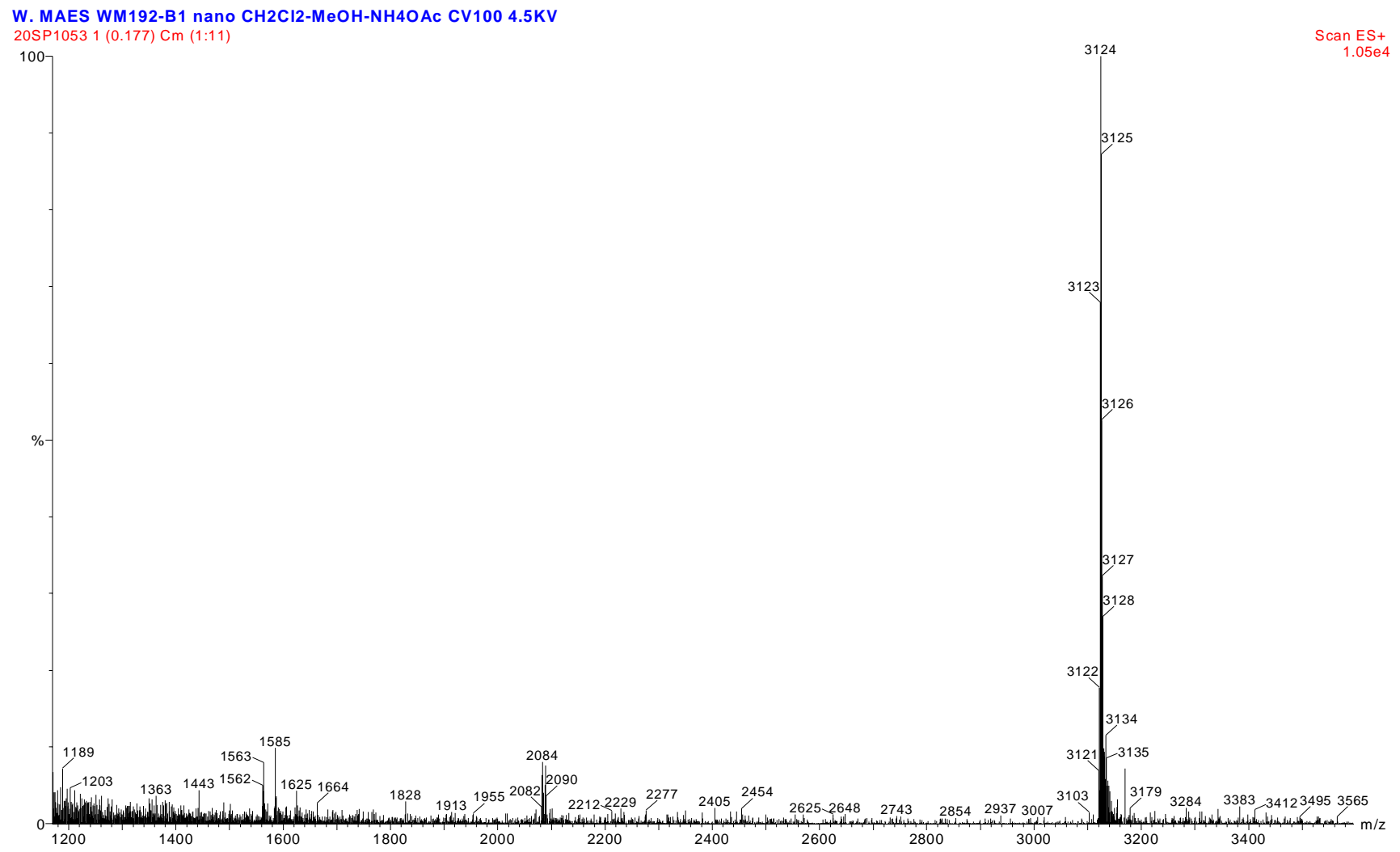

* $\mathrm{G}_{2}$-dendron 7c (MALDI, BMPM): 


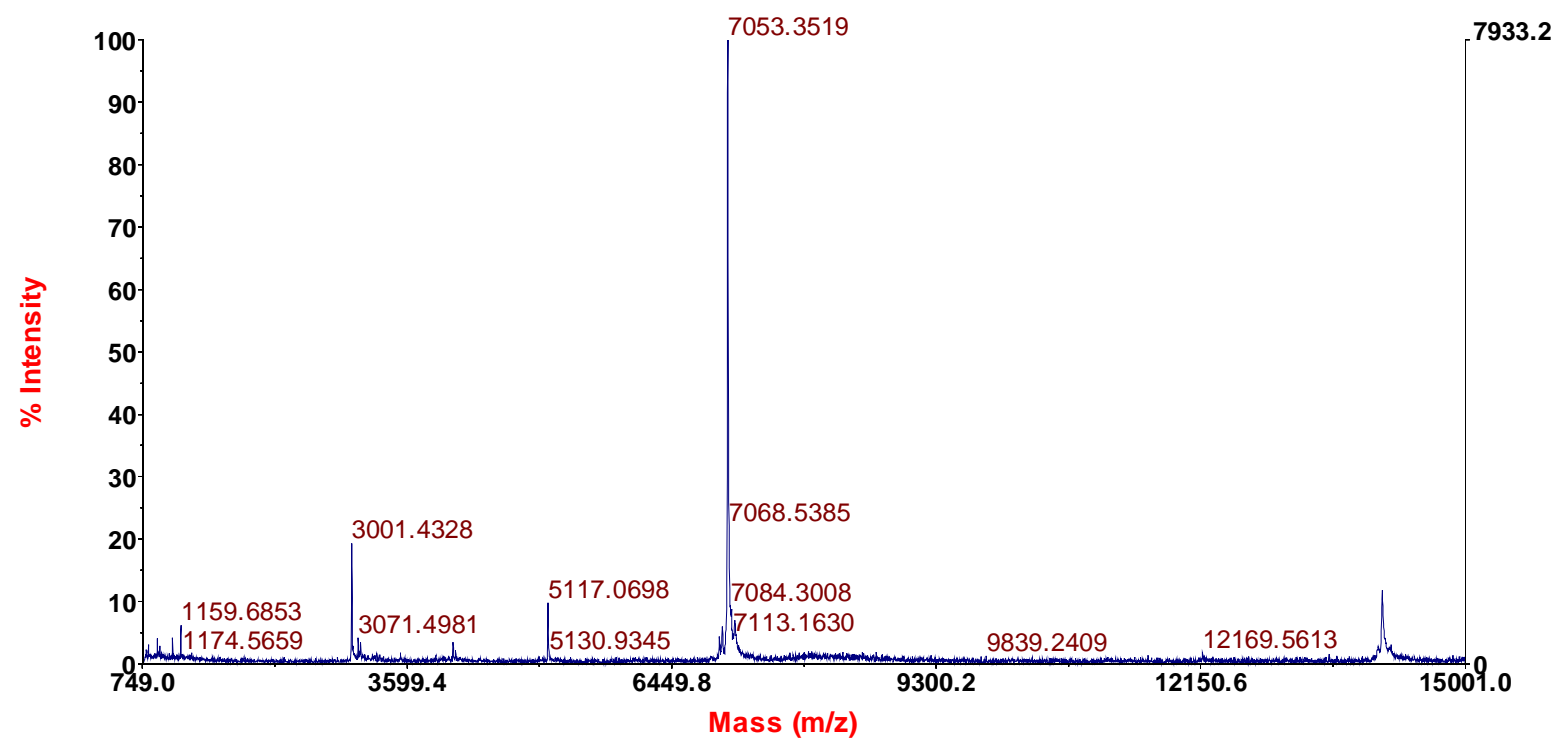

\section{* $\mathrm{G}_{0}$-dendrimer 11a (ESI):}

Smeets SSCV53 20/ ul/min MeOH-CH2Cl2 50-50 cone 200V met NH4OAc 20SP438 1 (0.193) Cm (1:11)

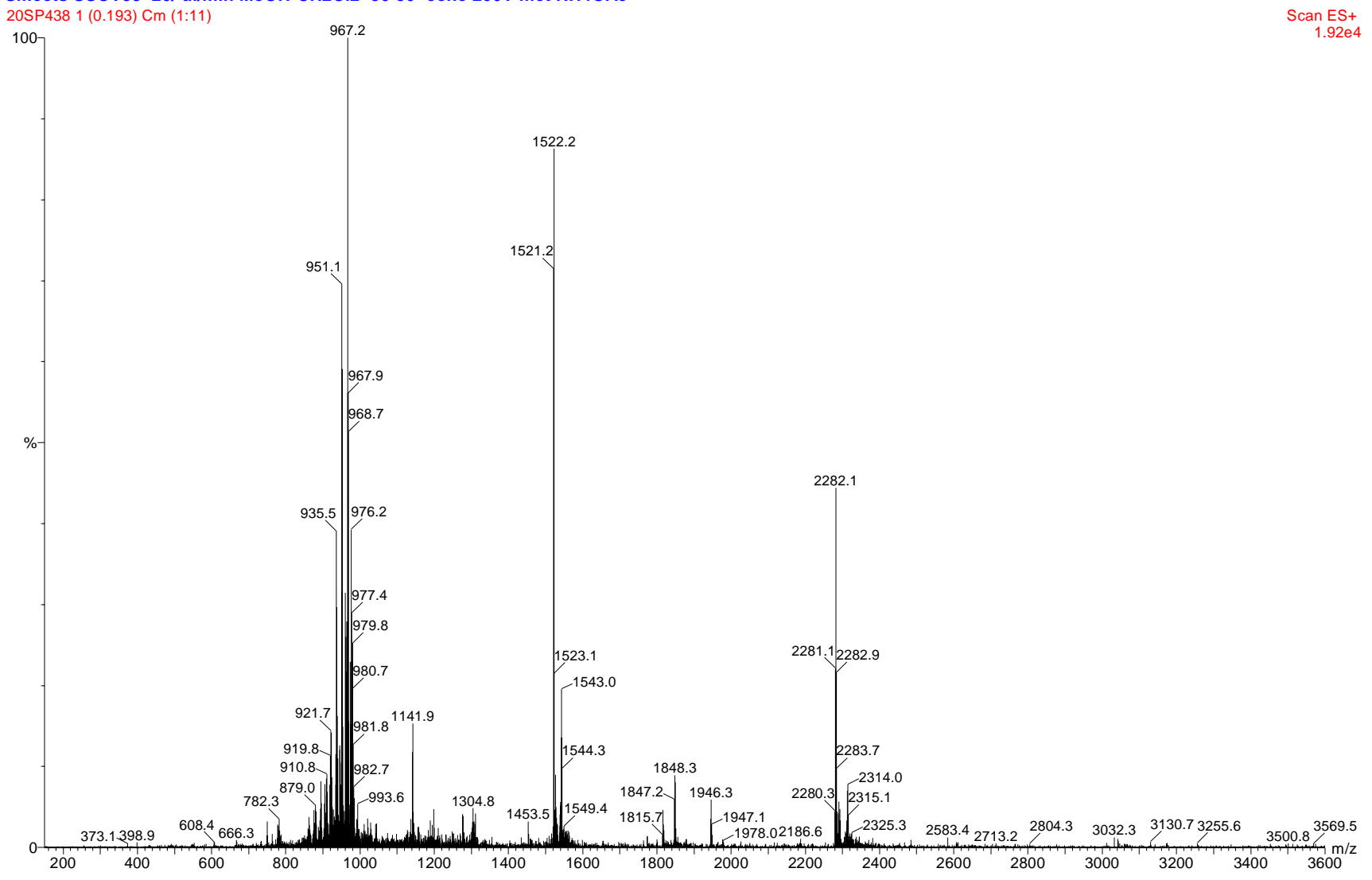

* $\mathrm{G}_{1}$-dendrimer 12 (MALDI, BMPM): 


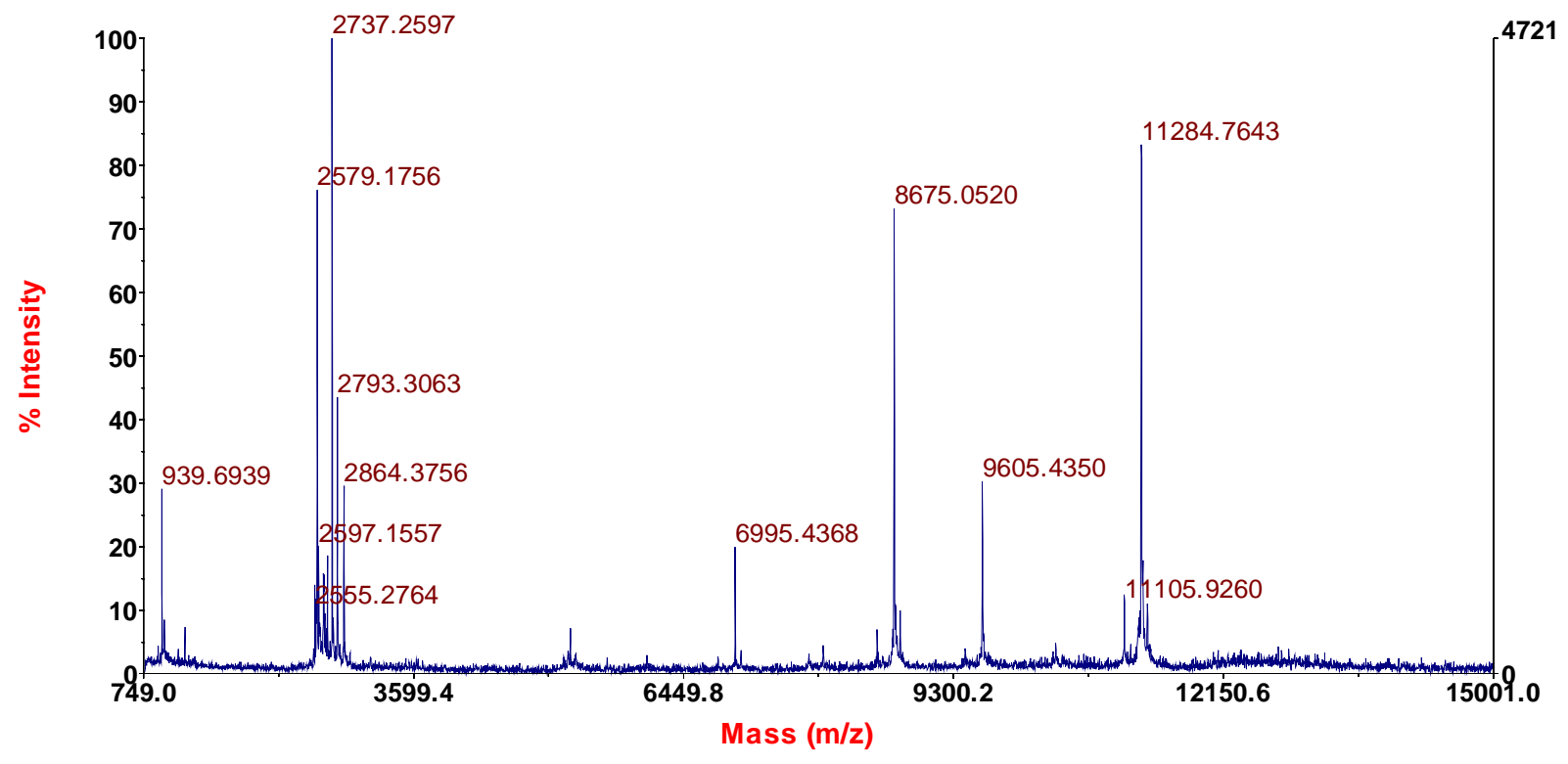




\section{Selected GPC Spectra}

* $\mathrm{G}_{1}$-dendron $\mathbf{6 c}$ (red, solid line) and $\mathrm{G}_{2}$-dendron $\mathbf{7 b}$ (black, dashed line):

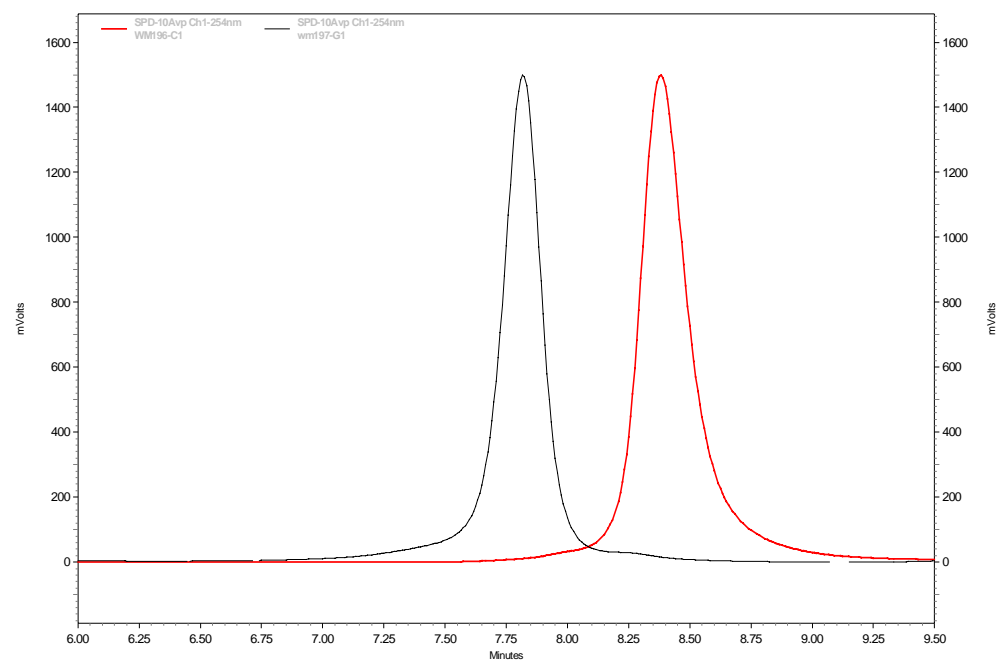




\section{Selected UV-vis Spectra}

* $\mathrm{G}_{2}$-dendron $7 \mathbf{b}\left(\mathrm{M}_{1}=\mathrm{Pt}, \mathrm{M}_{2}=\mathrm{Zn}, \mathrm{M}_{3}=\mathrm{H}, \mathrm{H}\right)$ :

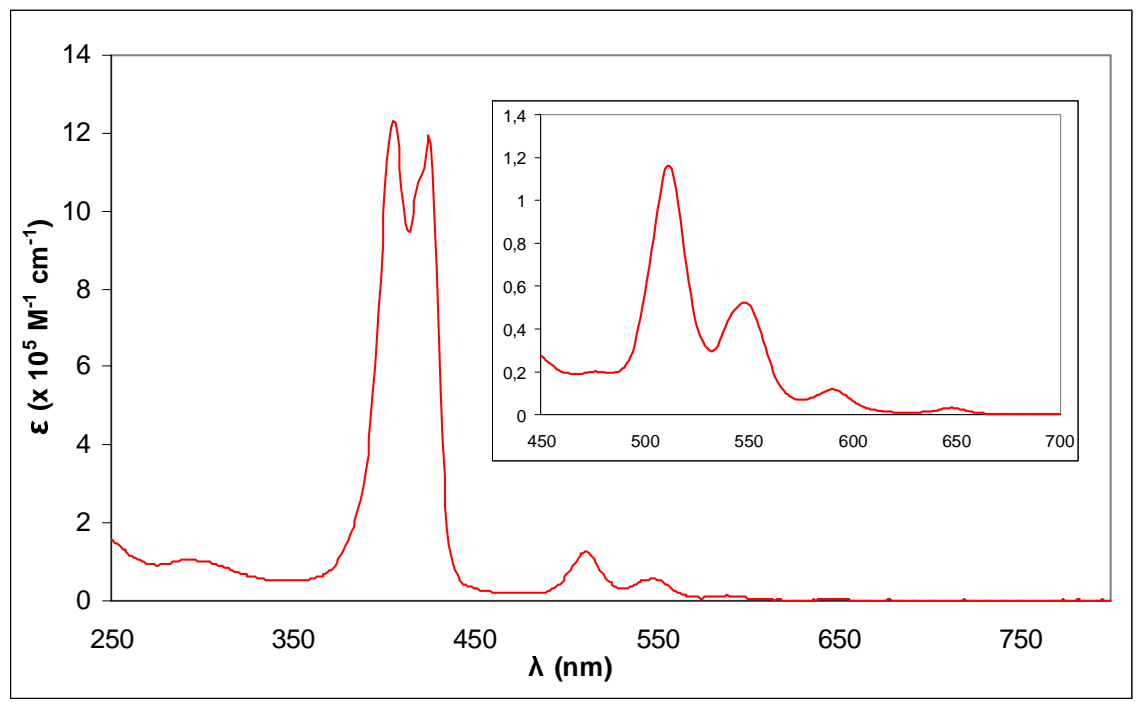

* $\mathrm{G}_{2}$-dendron 7c $\left(\mathrm{M}_{1}=\mathrm{Pt}, \mathrm{M}_{2}=\mathrm{Zn}, \mathrm{M}_{3}=\mathrm{Ni}\right)$ :

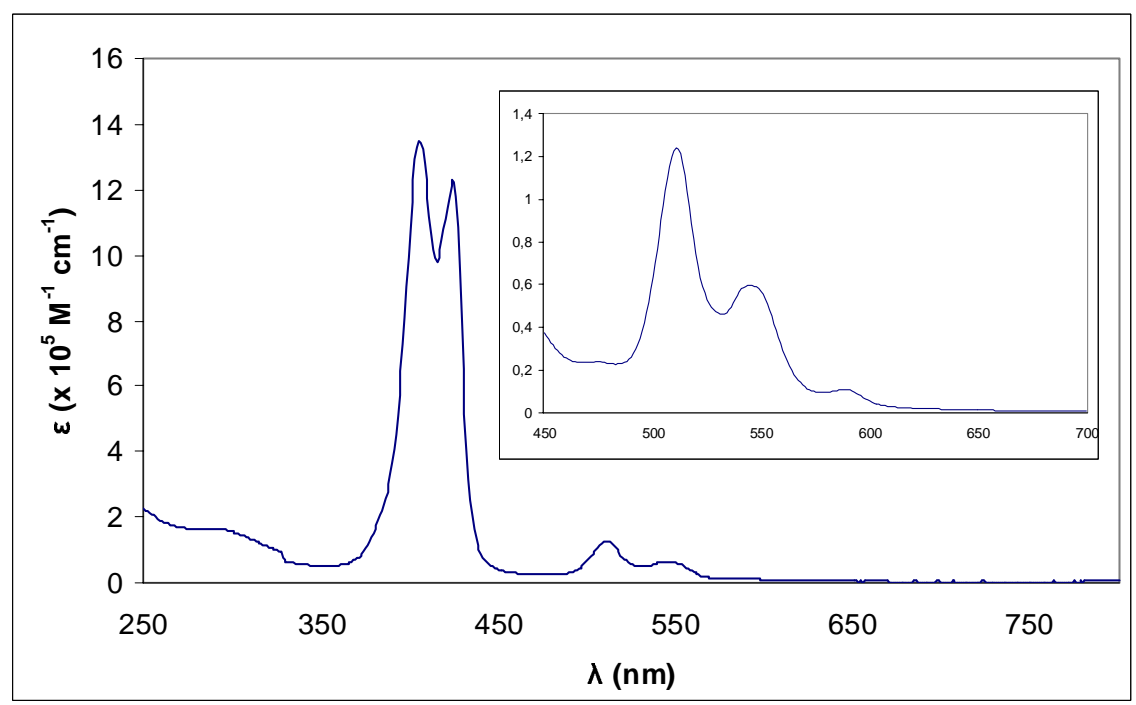


* $\mathrm{G}_{0}$-dendrimers 11a $\left(\mathrm{M}_{1}=\mathrm{M}_{4}=\mathrm{H}, \mathrm{H}\right.$; green) and 11b $\left(\mathrm{M}_{1}=\mathrm{Zn}, \mathrm{M}_{4}=\mathrm{H}, \mathrm{H}\right.$; blue): (a) full spectrum, (b) enlarged Q-band region:
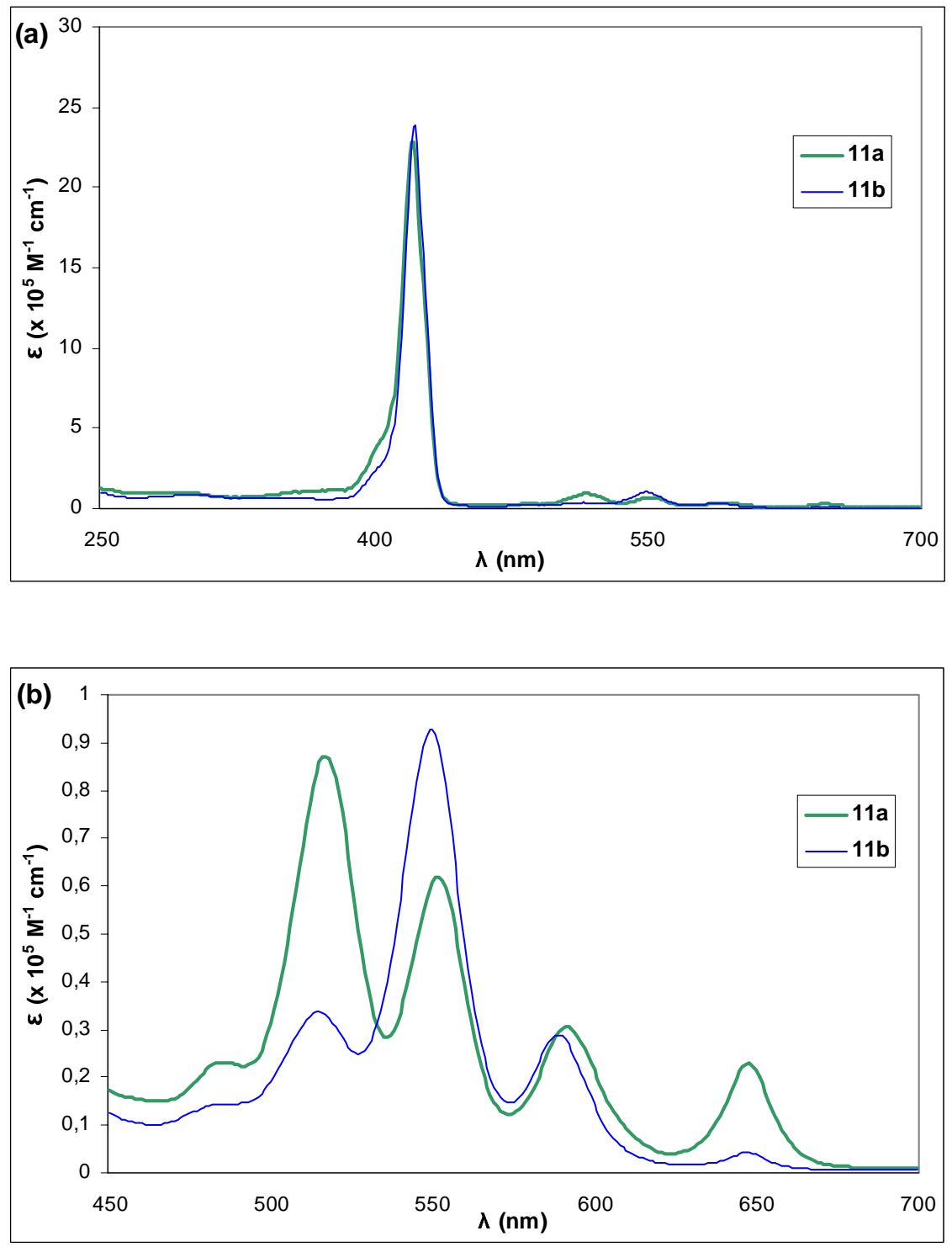